\author{
Aus dem Fachbereich Medizin \\ der Johann Wolfgang Goethe-Universität \\ Frankfurt am Main
}

betreut am

Zentrum der Chirurgie

Klinik für Thorax-, Herz- und Thorakale Gefäßchirurgie

Direktor: Herr Prof. Dr. Thomas Walther

\title{
Extracorporeal Life Support in Kombination mit IABP. Behandlungsmöglichkeiten und Grenzen einer supportiven Therapie bei kardialem Versagen. Retrospektive Analyse von 118 Patienten.
}

\author{
Dissertation \\ zur Erlangung des Doktorgrades der Medizin \\ des Fachbereichs Medizin \\ der Johann Wolfgang Goethe-Universität \\ Frankfurt am Main
}

vorgelegt von

Franziska Désirée Erfle

aus Heidelberg

Frankfurt am Main, 2020 
Dekan:

Prof. Dr. Stefan Zeuzem

Referent:

Prof. Dr. Nestoras Papadopoulos

Korreferent/in:

Prof. Dr. Udo Rolle

Tag der mündlichen Prüfung:

26.05.2021 


\section{Inhaltsverzeichnis}

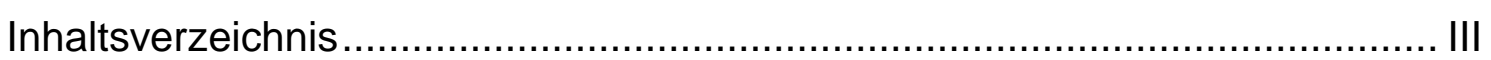

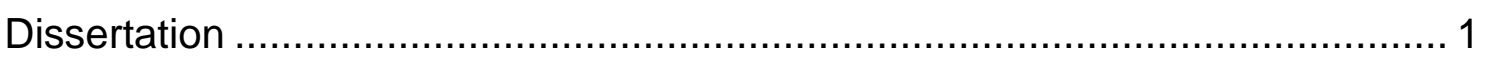

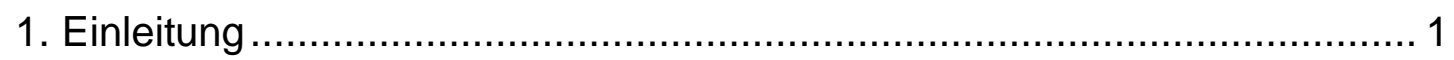

1.1 Grundprinzipien und Einsatzgebiet des Extracorporeal Life Support

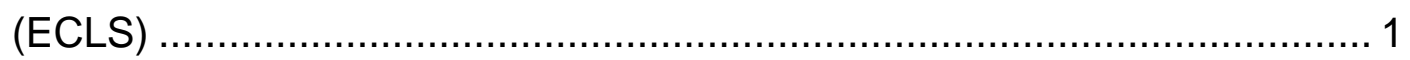

1.2 Etablierung der Extracorporeal Life Support - Systeme an der Universitätsklinik Frankfurt am Main ..................................................... 1

1.3 Funktionsweise des Extracorporeal Life Support ............................... 1

1.4 Aufbau des Extracorporeal Life Support - Systems ............................ 2

1.4.1 Funktionsweise der Extracorporeal Life Support - Einheit .............. 3

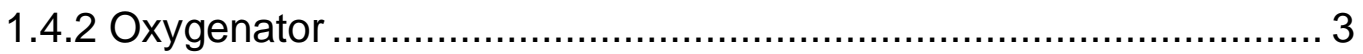

1.4.3 Wärmeaustauscher (Heater Unit) …………….......................... 4

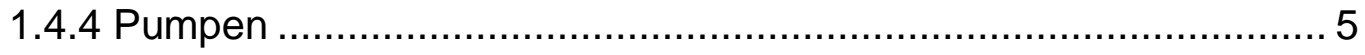

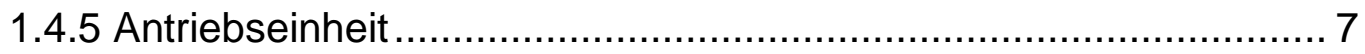

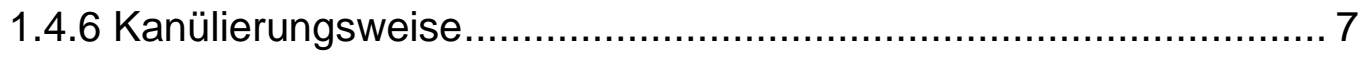

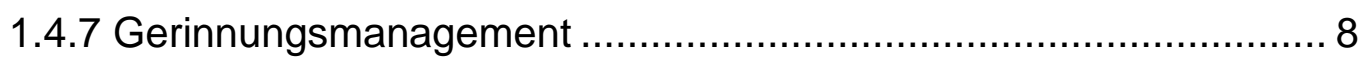

1.5 Indikationsstellung für die Implantation eines Extracorporeal Life

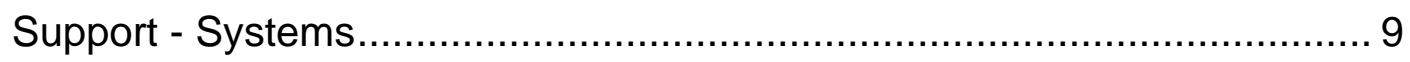

1.5.1 Indikationen bei Lungenversagen:............................................ 9

1.5.2 Indikationen bei Herzversagen ............................................... 10

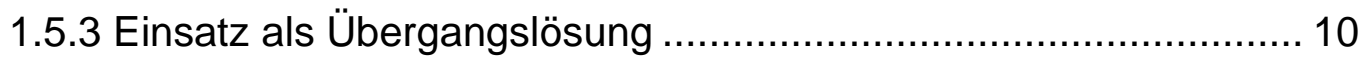

1.5.4 Kontraindikationen für die Extracorporeal Life Support - Therapie 10

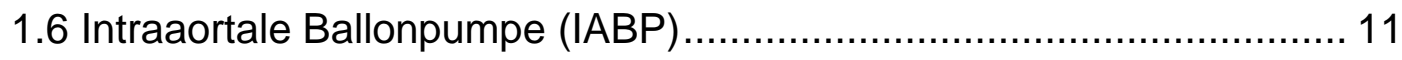

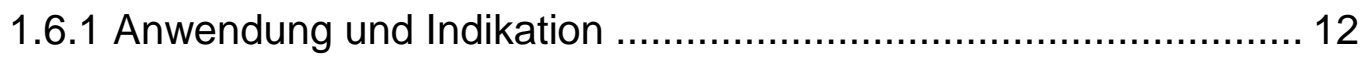



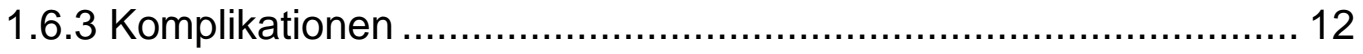

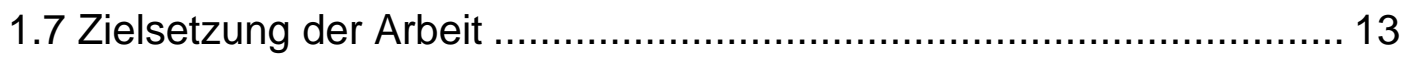




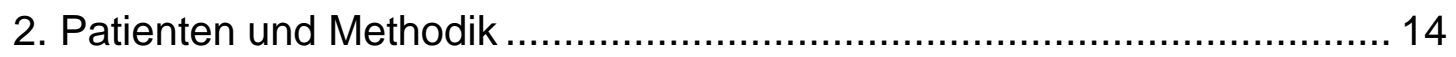

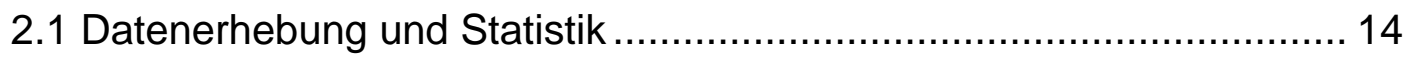



2.2.1 Risikoprofil der Gesamtpopulation ............................................. 14

2.2.2 Rahmenbedingungen der Implantation ....................................... 16

2.2.3 Operationen des Gesamtkollektivs ........................................... 17

2.3 Monitoring unter laufender Extracorporeal Life Support -Therapie...... 17

2.4 Procedere auf der Intensivstation................................................... 17

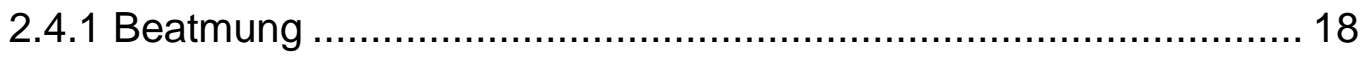

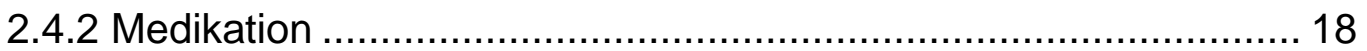

2.4.3 Entwöhnung von der Extracorporeal Life Support - Therapie

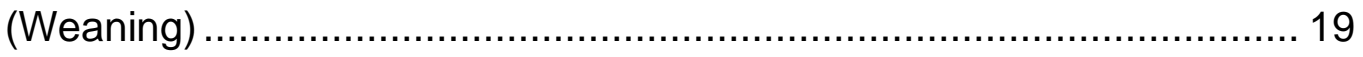

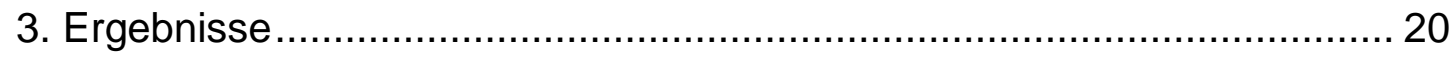

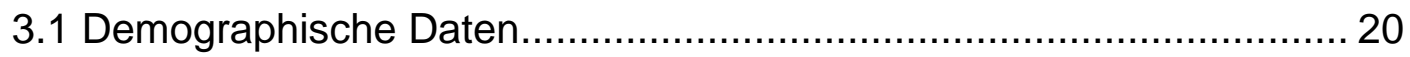

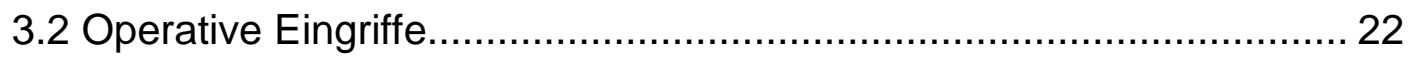

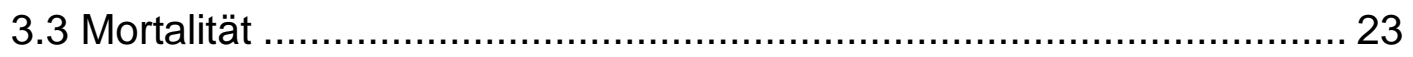

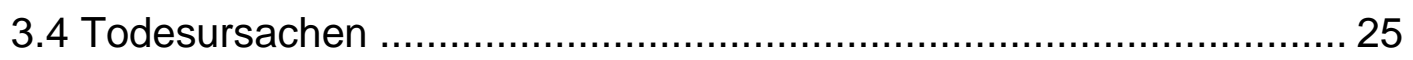

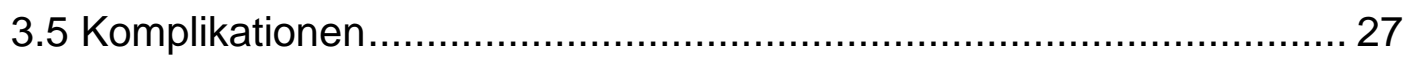

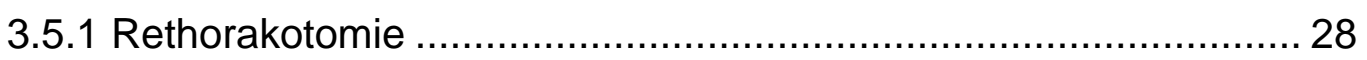

3.5.2 Blutung an der Kanülierungsstelle ........................................... 28





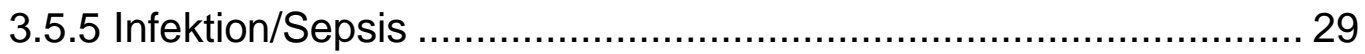

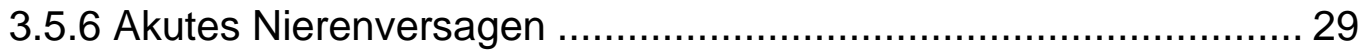

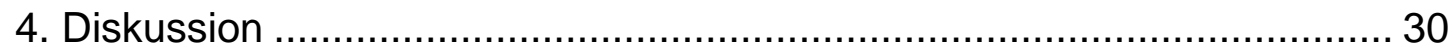

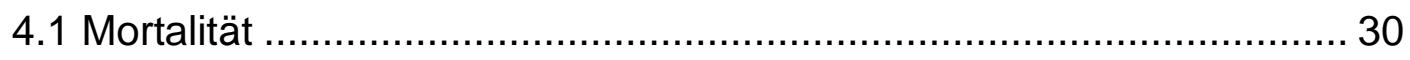

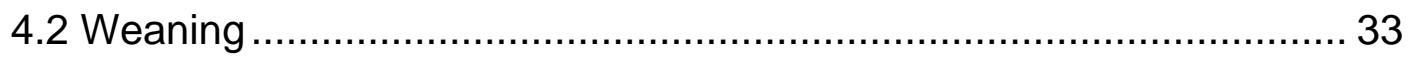


4.3 Risikofaktoren

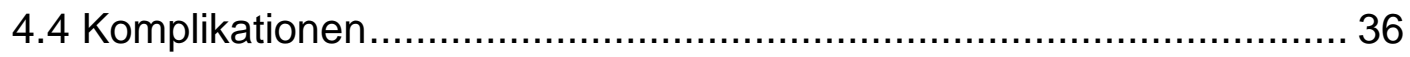



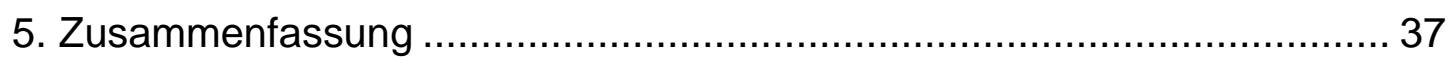

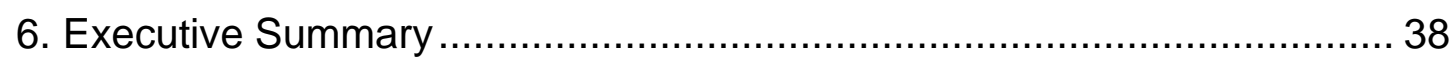

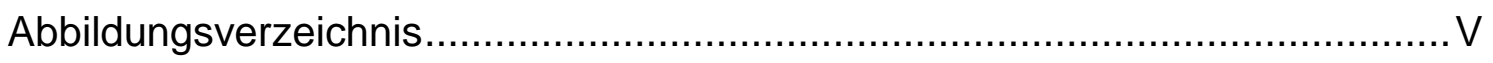

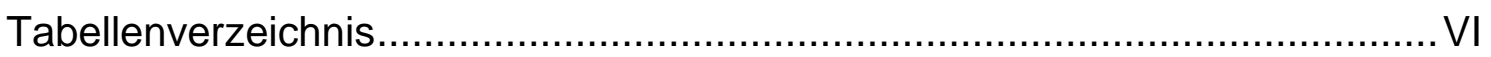

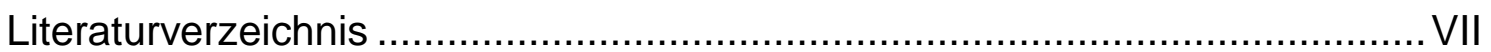

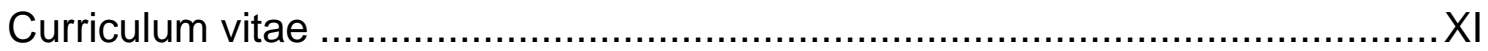

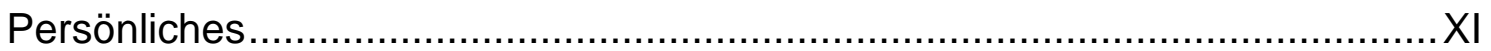

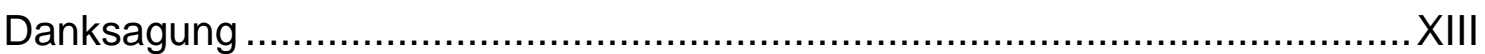

Schriftliche Erklärung …....................................................................... XIV 


\section{Dissertation}

\section{Einleitung}

\subsection{Grundprinzipien und Einsatzgebiet des Extracorporeal Life Support}

(ECLS)

Bei Patienten mit durch konservative Maßnahmen nicht beherrschbarem kardiogenem Schock, nach Herz-Kreislaufstillstand oder bei perioperativem Kreislaufversagen nach herzchirurgischem Eingriff werden ECLS-Systeme notfallmäßig zur Aufrechterhaltung des Blutkreislaufes eingesetzt. Es handelt sich um eine Modifikation der operativen Herz-Lungen-Maschine, wobei der Anschluss über periphere Gefäße erfolgt. ECLS-Systeme ermöglichen ebenfalls die externe Oxygenierung und die Fortführung des Herzkreislaufs am stillgelegten Herzen. Der Vorteil der ECLS-Systeme besteht darin, dass sie deutlich länger im Einsatz bleiben können als herkömmliche Rollerpumpensysteme, die Anwendung bei der Herz-Lungen-Maschine finden, und sich in der dadurch gewonnenen Zeit Herz und/oder Lunge erholen können. Dies liegt am Einsatz einer Zentrifugalpumpe, welche bezüglich des Auftretens einer Hämolyse deutlich von Vorteil ist. ${ }^{1}$

\subsection{Etablierung der Extracorporeal Life Support - Systeme an der}

\section{Universitätsklinik Frankfurt am Main}

Seit dem Jahr 2000 entwickelt sich die ECLS-Infrastruktur an der Klinik für Thorax-, Herz-, und thorakale Gefäßchirurgie Frankfurt am Main. Bis 2007 gab es zwei Pumpeinheiten, seit 2007 stehen bereits fünf zur Verfügung, allesamt bezogen von der Firma Life Systems (Medizintechnik-Service GmbH, Mönchengladbach).

\subsection{Funktionsweise des Extracorporeal Life Support}

Das sauerstoffarme, venöse Blut wird mittels einer Pumpe über ein venöses Schlauchsystem durch einen mit Heparin und Albumin beschichteten Oxygenator gepumpt. In diesem wird das Kohlenstoffdioxid eliminiert und das Blut wieder mit Sauerstoff angereichert. Der Rückfluss in den Körper kann über ein arterielles Schlauchsystem entweder in eine Arterie (veno-arteriell) oder in eine Vene (veno- 
venös) erfolgen. Die veno-venöse Variante kommt bei Patienten mit primär respiratorischem Versagen zum Einsatz, die noch eine adäquate Herzfunktion vorweisen können und wird ECMO (Extracorporeal Membrane Oxygenation) genannt. Bei primärem Kreislaufversagen oder respiratorischem und kardialem Versagen wird der in dieser Arbeit untersuchte veno-arterielle Zugang verwendet. $^{2}$ Zu diesem mehr unter Kanülierungsweise (Kapitel 1.4.5). Bei der Variante mit veno-arterieller Kanülierung spricht man von ECLS-Therapie (Extracorporeal Life Support).

\subsection{Aufbau des Extracorporeal Life Support - Systems}

In Abbildung 1 ist der Aufbau des verwendeten ECLS - Systems schematisch dargestellt.

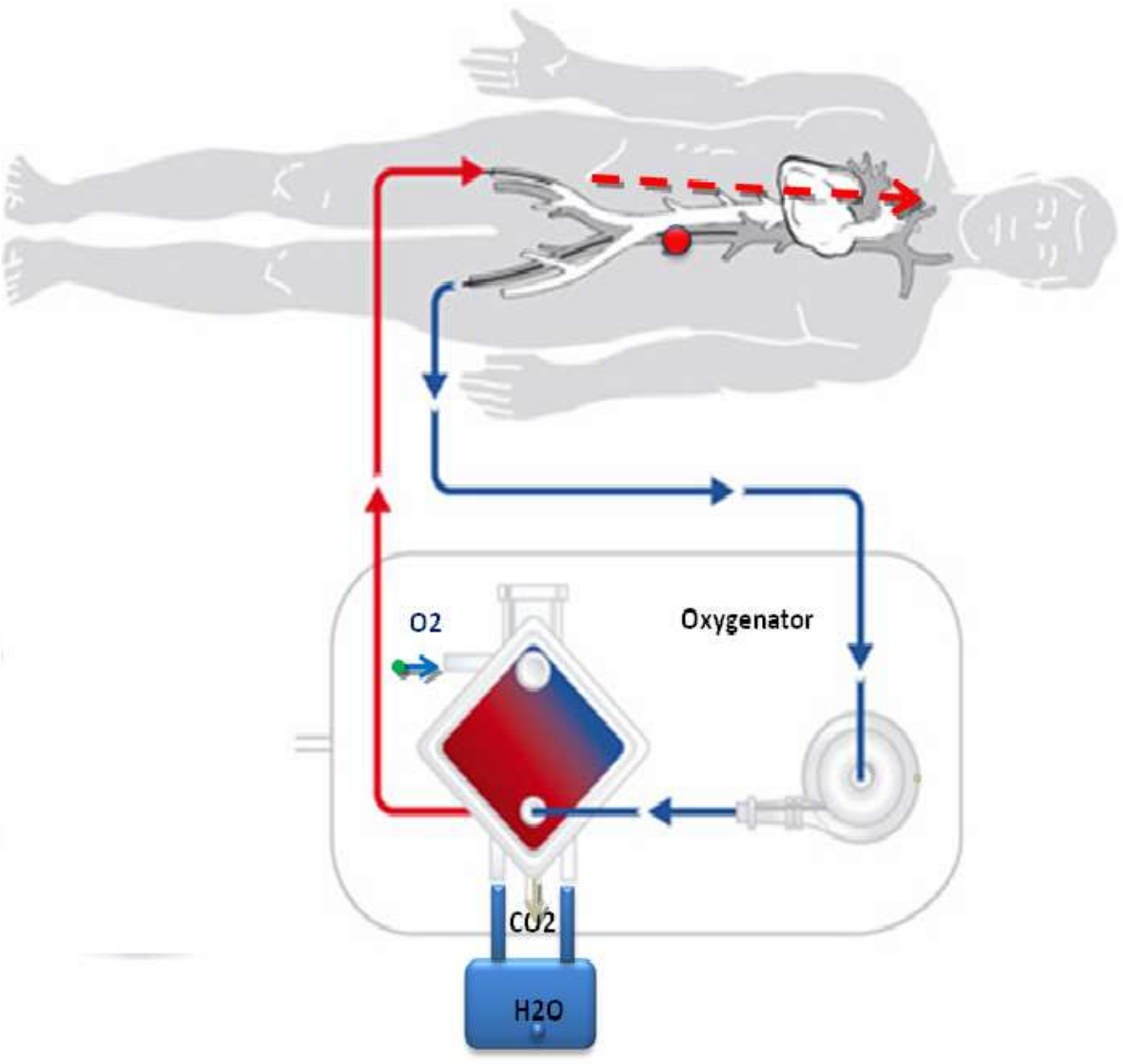

Abbildung 1: Schematische Darstellung des ECLS - Systems (mit freundlicher Genehmigung der Firma Maquet ()) 


\subsubsection{Funktionsweise der Extracorporeal Life Support - Einheit}

Das im Universitätsklinikum Frankfurt verwendete ECLS-System besteht aus Oxygenator, Zentrifugalpumpe und einem Schlauchsystem. Das Schlauchsystem besteht wiederum aus einer venösen Kanüle, über welche das Blut aus dem Körper in das System fließt, und einer arteriellen Kanüle, die das mit Sauerstoff angereicherte Blut zum Körper zurücktransportiert. Das Schlauchsystem ist heparinbeschichtet, um die Blutgerinnung aufgrund des Fremdoberflächenkontakts durch die Nachbildung menschlichen Gewebes zu verhindern (BIOLINE-Beschichtung) ${ }^{3}$. Es ist für einen vierzehntägigen Einsatz vorgesehen und kann somit wesentlich länger in Betrieb genommen werden, als eine ausschließlich im operativen Setting verwendete Herz-Lungen-Maschine, da durch den Einsatz der Zentrifugalpumpe das Risiko des Auftretens einer Hämolyse deutlich verringert ist.

\subsubsection{Oxygenator}

Der Oxygenator reichert das durch das System fließende Blut mit Sauerstoff an und ersetzt somit die Lunge. Die gasführenden Fasern der Polymethylpenten (PMP)-Fasern geben den Sauerstoff via Diffusion an das Blut ab und nehmen Kohlendioxid auf (siehe Abbildung 2). Treibende Kraft ist hierbei die Partialdruckdifferenz. Der Partialdruck für Sauerstoff beträgt in der Gasphase etwa $760 \mathrm{mmHg}$. Im Blut beträgt der Partialdruck für Sauerstoff in gemischtvenösem Blut etwa $40 \mathrm{mmHg}$ und für Kohlendioxid zwischen 35 und 45 $\mathrm{mmHg}$. In der Gasphase befindet sich kein Kohlendioxid. Die Diffusion über die Membran erfolgt so vom Ort des höheren Partialdrucks zum Ort des niedrigeren. Obwohl die Oberfläche der Membranoxygenatoren nur circa 1/10 der Gasaustauschfläche der Lunge beträgt, wird der Körper ausreichend oxygeniert. Dies liegt zum einen an der längeren Kontaktzeit zwischen Blut und Gas im Oxygenator und zum anderen am höheren Partialdruckkonzentrationsgefälle, wie durch das Fick'sche Gesetz erkenntlich wird:

$$
V_{O_{2}}=\frac{P_{1}-P_{2}}{\mathrm{~L}} K F
$$

\footnotetext{
$\mathrm{V}_{\mathrm{O}_{2}} \quad$ ausgetauschte $\mathrm{O}_{2}$-Menge/ Zeiteinheit

$\mathrm{P}_{1}-\mathrm{P}_{2} \quad$ Partialdruckdifferenz

KF Körperoberfläche

L Schichtdicke
} 


\section{Diffusionsmembran}

\section{Polymethylpenten (PMP) Membran}

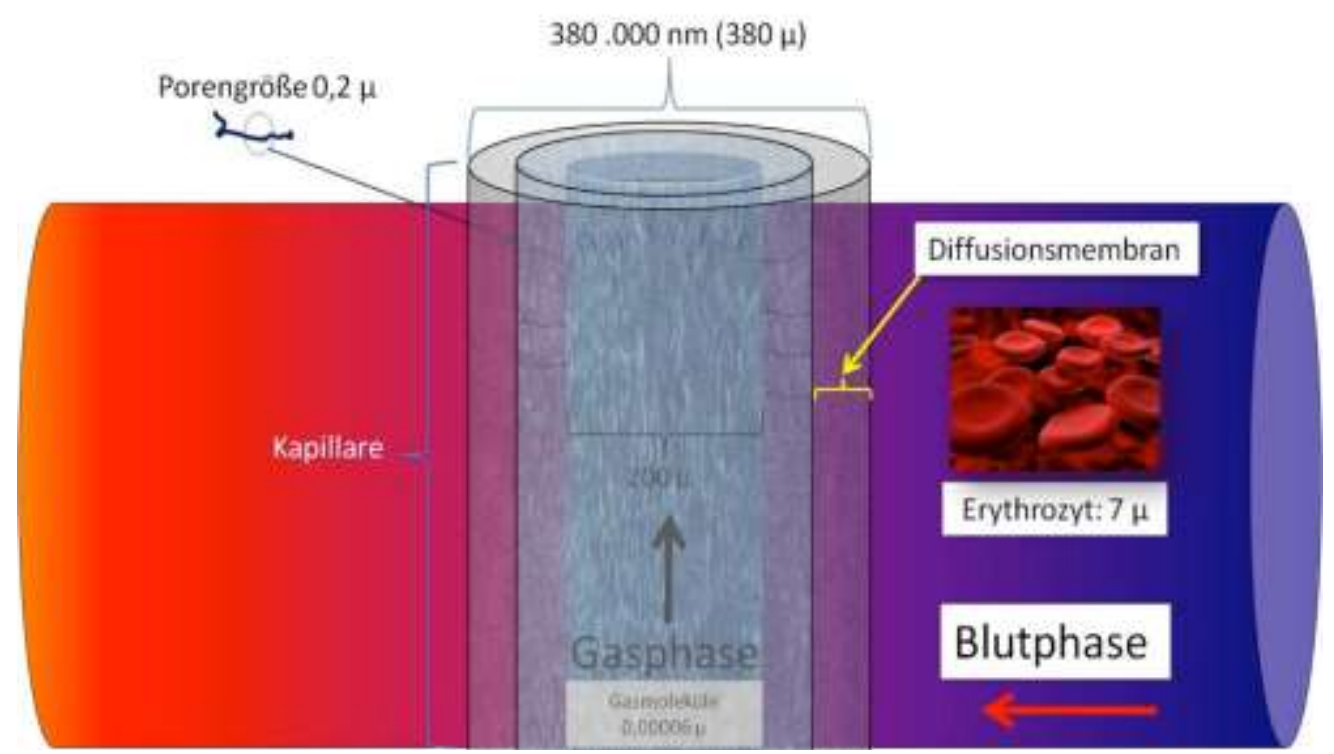

Abbildung 2: Schematischer Aufbau einer Diffusionsmembran (mit freundlicher Genehmigung der Firma Maquet (C)

Durch die Benutzung von Diffusionsmembranen lässt sich im Gegensatz zu mikroporösen Membranfasern, wie sie früher verwendet wurden, ein Austritt von Plasma bei Langzeiteinsatz der ECLS verhindern. Gleichzeitig ist die homogene PMP-Oberfläche blutschonender. Allerdings können volatile Anästhetika aufgrund ihrer Unfähigkeit die PMP-Membran zu passieren nur bei mikroporösen Membranen verwendet werden.

Die Gabe von Blutprodukten und Ablagerungen von Fibrin können einen vorzeitigen Systemwechsel erforderlich machen, da durch sie die Diffusionsstrecke verlängert wird. ${ }^{4,5}$

\subsubsection{Wärmeaustauscher (Heater Unit)}

Durch den in den Oxygenator integrierten Wärmeaustauscher wird eine Modulation der Temperatur des Patienten möglich und so dessen Auskühlen verhindert. Die in Abbildung 3 dargestellte Heater Unit 35 (HU 35) besitzt zum Beispiel einen 1,5 Liter fassenden Wassertank. Das Wasser wird von einem Motor mit der gewünschten Temperatur durch den Oxygenator gepumpt, wärmt oder kühlt dementsprechend das hindurchfließende Blut. Der mögliche Temperaturbereich liegt zwischen 33 und $39^{\circ} \mathrm{C}$. 
Bei Senkungsbedarf, im Fall, dass der Patient Fieber entwickelt, kann die Einheit temporär abgeschaltet werden. Da sie allerdings keinen Akku besitzt, kann sie nicht während eines Transports eingesetzt werden.

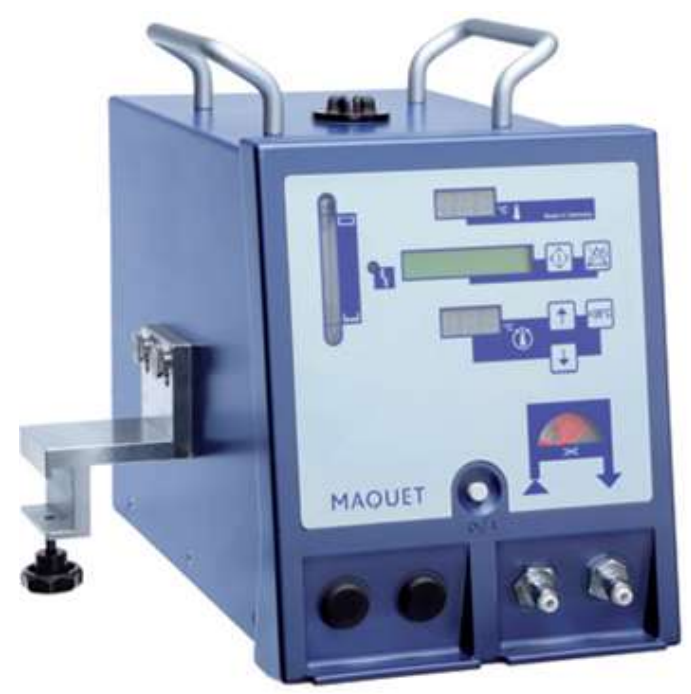

Abbildung 3: Vorderseite der HU 35 (mit freundlicher Genehmigung der Firma Maquet $\odot$ )

\subsubsection{Pumpen}

Grundsätzlich lassen sich zwei Arten von Pumpen unterscheiden, die in der Herzchirurgie Anwendung finden. Zum einen gibt es Verdrängungspumpen (Rollerpumpen), welche durch die Bewegung von Rollen und die damit verbundene Kompression im Schlauchsystem für die Beförderung des Blutes sorgen. Diese finden vorwiegend in konventionellen Herz-Lungen-Maschinen Verwendung. Die mechanische Verformung eines sich in der Pumpe befindlichen Schlauches sorgt dafür, dass das Blut durch diesen hindurchgedrückt "verdrängt"- wird. Aus diesem Grunde werden diese Pumpen auch als "Schlauchquetschpumpen“ bezeichnet. Daher hat der Schlauch durch die starke mechanische Beanspruchung eine verkürzte Lebensdauer. Außerdem kommt es dadurch auch zu Abrieb am Schlauchsystem, was auf Dauer eine Verunreinigung des Blutes sowie eine ausgeprägte Hämolyse zur Folge hat. ${ }^{6}$

Zum anderen gibt es Zentrifugalpumpen, auch Kreiselpumpen genannt, welche die Blutflüssigkeit dadurch in Bewegung setzen, dass sie durch Rotation eines Laufrads die durch die Zentrifugalkraft entstehende kinetische Energie auf das Blut übertragen (Abbildung 4). 
Durch die Bewegungsenergie des Bluts kommt es zu einer Druckerhöhung innerhalb der Pumpe und die Flüssigkeit wird in das nachgeschaltete Druckrohr gepresst. Die Zentrifugalpumpe stellt durch vermindertes Auftreten von Hämolyse die schonendere Variante dar. Zentrifugalpumpen müssen vor Inbetriebnahme mit Flüssigkeit gefüllt sein („Priming“). Um ein Zusammenbrechen des Pumpenbetriebs zu unterbinden, muss darauf geachtet werden, dass währenddessen nicht mehr als 8-10 Volumenprozent Luft in die Saugleitung eindringen. Die Zentrifugalpumpe hat im Vergleich $\mathrm{zu}$ den Verdrängungspumpen einige weitere Vorteile. Hierzu gehören weniger Turbulenzen, die Vermeidung einer Überhitzung der Pumpe durch Blut und Reibung und ein vom Gefäßwiderstand geringfügiger abhängiger Pumpenfluss. Die erforderliche Flussrate von 1-4 l/min bei 1000-4000 Umdrehungen kann von beiden Pumpenarten erreicht werden. Die maximale Flussrate beträgt etwa 50$70 \mathrm{ml} / \mathrm{kg} / \mathrm{min}$ und wird zunächst für 24 bis 72 Stunden aufrechterhalten.

Bei der in der Herzchirurgie des Frankfurter Uniklinikums bevorzugt verwendeten Zentrifugalpumpe ROTAFLOW RF-32@ handelt es sich um eine sogenannte Radialpumpe, in deren Innerem der Fluss im rechten Winkel umgelenkt wird (Abbildung 4).

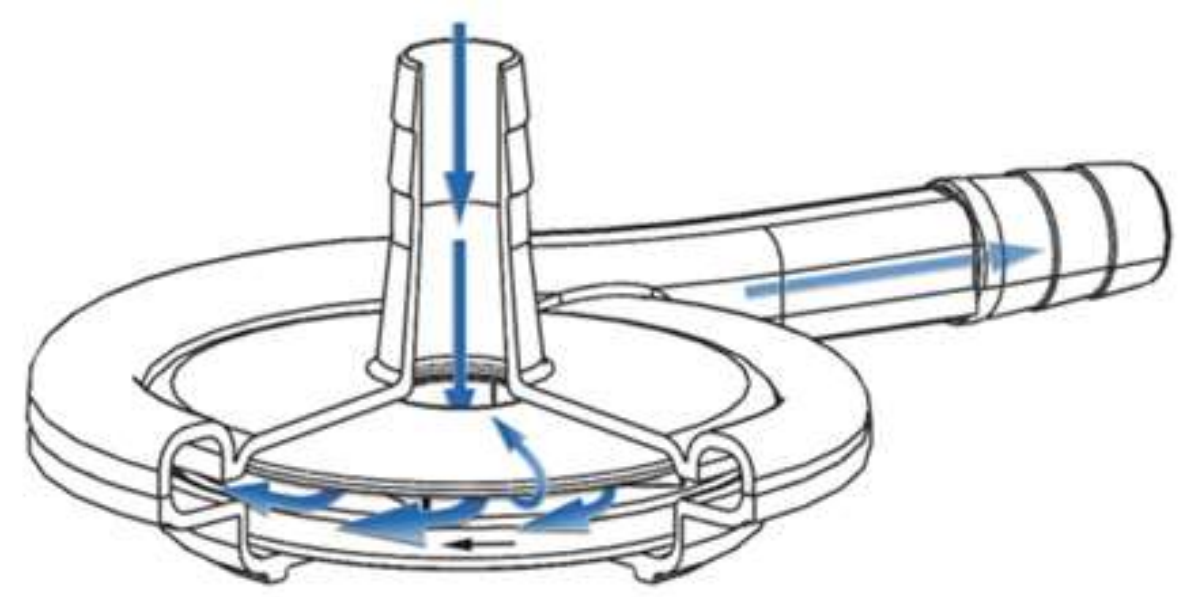

Abbildung 4: Funktionsprinzip einer ROTAFLOW @ Zentrifugalpumpe (mit freundlicher Genehmigung der Firma Maquet ( $)$ 


\subsubsection{Antriebseinheit}

Die Antriebseinheit besteht aus einer Pumpe und einer Konsole, an der sich die Umdrehungszahl der Blutpumpe einstellen lässt. Die Konsole verfügt außerdem über einen Akku, der circa 1,5 Stunden lang netzunabhängig arbeiten kann. Bei Versagen der Antriebseinheit kann im Notfall eine Handkurbel manuell betätigt werden, um den Blutfluss aufrecht zu erhalten.

\subsubsection{Kanülierungsweise}

Indem der arterielle Schenkel mittels Sternotomie offen-chirurgisch an die Aorta ascendens angeschlossen wird, erfolgt bei ECLS-Therapie die Kanülierung zentral. Alternativ kann man zur Vermeidung einer Mediastinitis die arterielle Kanüle ebenfalls direkt oder selten perkutan mittels Seldinger-Technik mit der Arteria subclavia verbinden. Diese Anwendung, welche perkutan relativ schnell zu etablieren ist, kommt vor allem bei Patienten mit laufender kardiopulmonaler Reanimation (CPR) zum Einsatz (häufiger jedoch ist der Gebrauch der Arteria femoralis, s.u.). Bei Patienten mit kleinem Gefäßdurchmesser entscheidet man sich häufig für die direkte Kanülierung. Durch die Punktion einer Arterie besteht logischerweise ein höheres Blutungsrisiko als bei einer veno-venösen Kanülierung. Der Vorteil der Kanülierung der rechten A. subclavia besteht in der Vermeidung einer massiven Nachlasterhöhung wie sie bei Kanülierung der Femoralgefäße der Fall ist. Bei der peripheren Kanülierung wird der arterielle Schenkel ebenfalls entweder direkt oder via Seldinger-Technik perkutan an die A. femoralis angeschlossen. Es gibt neuerdings jedoch Möglichkeiten vor Anlage der Kanüle mittels Proglide-Verfahren, um eine mögliche Explantation der Kanüle ohne chirurgische Revision durchführen zu können. ${ }^{7}$ Die venöse Komponente wird mit der ipsi- oder auch kontralateralen Vena femoralis verbunden. Durch die veno-arterielle Kanülierung kann nicht nur denjenigen Patienten mit respiratorischem Versagen geholfen werden, sondern auch Patienten bei denen sowohl Lunge als auch das Herz deutlich kompromittiert sind. 


\subsubsection{Gerinnungsmanagement}

Für ein reibungsloses Funktionieren der ECLS-Therapie ist ein genau abgestimmtes Gerinnungsmanagement von großer Bedeutung. Die unphysiologischen Oberflächen der ECLS aktivieren sekundär das Gerinnungssystem, wodurch das Thromboserisiko steigt. Um dem entgegenzuwirken werden zum einen sowohl die Schlauchsysteme, als auch die Oxygenatoren mit Heparin beschichtet (BIOLINE-Beschichtung). In Frankfurt erhalten die Patienten zusätzlich eine Initialdosis von $5000 \mathrm{IE}$ Heparin als Bolus. Allerdings besteht durch die ECLS-Therapie nicht nur ein erhöhtes Thromboserisiko. Es können auch gefährliche Koagulopathien wie die Heparininduzierte Thrombozytopenie (HIT) auftreten, die durch erworbene Störungen der primären Hämostase, aber auch durch Hämodilution und einen erhöhten Verbrauch an Gerinnungsfaktoren verursacht sein können.8,9 Dennoch ist eine prophylaktische Antikoagulation nicht zu vernachlässigen. Hierzu verwendet man unfraktioniertes Heparin, mit welchem man eine etwa 1,5-2-fach erhöhte aktivierte partielle Thromboplastinzeit (aPTT, Normwert: 24,5-32,4 s) erreichen will. Die Ziel - pTT liegt hierbei bei ca. 50-60 s. Gleichzeitig lässt sich über diesen Wert die Gerinnung des Patienten überwachen. Das Heparin wird gewichtsadaptiert mit einer Konzentration von 100 oder $200 \mathrm{IE} / \mathrm{ml}$ über einen Perfusor kontinuierlich appliziert. Wie unschwer zu erkennen, begibt man sich bei dem Versuch eines adäquaten Gerinnungsmanagements häufig auf eine Gratwanderung zwischen der Verhinderung von Blutungskomplikationen bis hin zur Transfusionspflicht einerseits und der therapeutischen Antikoagulation andererseits, mit der man versucht Thrombosen, hervorgerufen durch den Fremdoberflächenkontakt zu vermeiden. Das Gerinnungsmanagement in Frankfurt für Patienten nach kardiochirurgischen Eingriffen mit extrakorporaler Zirkulation richtet sich nach einem bestimmten Algorithmus, der aufgrund der Erkenntnis entwickelt wurde, dass bei circa $20 \%$ dieses Patientenklientels eine klinisch relevante Blutungsneigung auftritt. ${ }^{10}$ Mit diesem Algorithmus wird die Transfusion von Plasmaprodukten sowie Gerinnungsfaktoren anhand diagnostischer Faktoren wie Blutgasanalyse, activated clotting time, aktivierte partielle Thromboplastinzeit (aPPT), dem Quick-Wert und der gesteuert. Die Werte der Blutgasanalyse wurden mithilfe eines Blutgasanalyse-Gerätes (ABL 800 FLEX Blutgasanalysator, Radiometer Copenhagen, Dänemark) erhoben. 


\subsection{Indikationsstellung für die Implantation eines Extracorporeal Life}

\section{Support - Systems}

Die im Jahr 1989 gegründete Extracorporeal Life Support Organization (ELSO) hat klare Leitlinien für die Verwendung der ECLS-Systeme aufgestellt, welche zuletzt im August 2015 aktualisiert wurden (Version v1.4). ${ }^{11}$ Laut diesen sind grundsätzliche ECLS-Indikationen schweres, unter konventioneller Therapie refraktäres Herz- und/oder Lungenversagen mit einem Mortalitätsrisiko von 80\%. Der ECLS - Einsatz ist ab einem 50\%-igen Mortalitätsrisiko in Erwägung zu ziehen. Dieses Risiko wird so genau wie möglich unter Betrachtung von Altersgruppe und Schwere des Organversagens bestimmt. Weitere Indikation für die Implantation eines ECLS-Systems ist die Unterstützung von Herz und Lunge während einer ausgedehnten bronchoalveolären Lavage, Operationen im Bereich des Mediastinums oder der Luftröhre oder der Verschluss der Koronarien während Eingriffen am Herzen.

\subsubsection{Indikationen bei Lungenversagen:}

Indikationen bei Lungenversagen gemäß ELSO ${ }^{12}$ sind:

- Erwägung ab einem 50\%-igem Mortalitätsrisiko: $\mathrm{PaO}_{2} / \mathrm{FiO}_{2}<150$ mit $\mathrm{FiO}_{2}$ $>90 \%$ und/oder Murray score $2-3$, AOI score $^{\mathrm{b}}>60$ oder APPS score ${ }^{\mathrm{c}}$

- immer indiziert bei $\mathrm{PaO}_{2} / \mathrm{FiO}_{2}<100$ bei $\mathrm{FiO}_{2}>90 \%$ und/ oder Murray score 3-4 trotz sechs Stunden langer optimaler Versorgung $(80 \%$ Mortalität $)^{13}$

- $\mathrm{CO}_{2}$ - Retention bei maschineller Beatmung trotz hohem Plateaudruck (Pplat): > $30 \mathrm{~cm} \mathrm{H} \mathrm{H}_{2} \mathrm{O}$

- schweres Atemnotsyndrom

- Intubationsbedarf bei Patienten, die auf der Liste für eine Lungentransplantation stehen

- akutes Herz- oder respiratorisches Versagen (Lungenembolie, verlegte Atemwege, Nichtansprechen auf optimale Versorgung)

\footnotetext{
a Murray Score: quantifiziert den Schweregrad eines Lungenversagens anhand von Röntgenbefund, Oxygenierungsindex, dem positiven endexspiratorischen Druck und der effektiven Compliance der Lunge ${ }^{13}$.

${ }^{\mathrm{b}}$ Der altersangepasste Oxygenierungsindex für Erwachsene (AOI) berechnet sich wie folgt: Pplat $\mathrm{x} \mathrm{FiO}_{2} \div$ $\mathrm{PaO}_{2}{ }^{14}$.

${ }^{c}$ Mit dem APPS Score (age, $\mathrm{PaO}_{2} / \mathrm{FiO}_{2}$, plateau pressure score), der 24 Stunden nach Beginn einer protektiven Beatmung bei Patienten mit ARDS bestimmt wird, lässt sich deren Prognose einschätzen ${ }^{15}$.
} 


\subsubsection{Indikationen bei Herzversagen}

- nicht ausreichende Gewebeperfusion, welche man trotz ausreichend intravasalem Volumen an Hypotension und sehr geringer Ejektionsfraktion erkennt

- persistierender Schock trotz konventioneller Therapie in Form von medikamentöser Kreislaufunterstützung und Volumengabe, sowie Einsatz einer IABP, sofern diese im Einzelfall zweckdienlich ist

- Myokardinfakt, Myokarditis, peripartale Kardiomyopathie, dekompensiertes chronisches Herzversagen, Postkardiotomiesyndrom

- septischer Schock (zählt nicht überall zu den Indikationen)

\subsubsection{Einsatz als Übergangslösung}

Das ECLS - System wird auch als Übergangslösung eingesetzt. Als Ziel ist zum einen die komplette Erholung nach Myokardinfarkt, Myokarditis oder konventionellen Herzoperationen zu nennen (Bridge-to-destination). Zum anderen wird das ECLS - System zur Überbrückung bis zum Erhalt einer Herztransplantation eingesetzt (Bridge-to-transplantation). Dies kommt vor allem bei nicht revaskularisierbaren Herzinfarkten und chronischem Herzversagen vor. Ein weiterer Einsatzbereich ist die Überbrückung bis zur Implantation eines Ventricular Assist Devices (VAD) oder eines Kunstherzens (Bridge-to-assist).

\subsubsection{Kontraindikationen für die Extracorporeal Life Support - Therapie}

Kontraindikationen für die ECLS-Therapie entstehen individuell durch genaues Abwägen des Verhältnisses von Risiko und Nutzen des Einsatzes und müssen deshalb immer relativ gesehen werden.

Hierzu gehören:

- Umstände, die mit einem normalen Leben nach der Beendigung der ECLS-Therapie nicht vereinbar sind

- bereits zuvor existierende Faktoren, welche die Lebensqualität beeinflussen werden

- das Alter des Patienten

- eine Aussichtslosigkeit der Therapie bei infauster Prognose für den Patienten 
Speziellen Kontraindikationen bei Lungenversagen

- intensive Beatmung für mindestens eine Woche

- medikamentöse Immunsuppression

Spezielle Kontraindikationen bei Herzversagen

- absolute Kontraindikationen

- therapieresistentes Herzversagen, wobei gleichzeitig kein anderer Weg, wie beispielsweise eine Transplantation oder der Einbau eines Ventricular Assist Devices eingeschlagen werden kann

- hohes Lebensalter

- chronisches Organversagen

- eingeschränkte Compliance

- lange kardiopulmonale Reanimation, während welcher die Gewebsperfusion nicht suffizient war

- relative Kontraindikationen

- die Kontraindikation für eine antikoagulative Therapie

- Übergewicht

- hohes Lebensalter

\subsection{Intraaortale Ballonpumpe (IABP)}

Die IABP hat sich seit ihrer klinischen Einführung im Jahr 1967 zu dem am häufigsten eingesetzten mechanischen Herzunterstützungssystem entwickelt. Sie dient als Hilfsmittel zur Unterstützung der Herztätigkeit mit dem Ziel ein Herzversagen zu vermeiden. Wie der Name schon sagt, wird die Pumpe nach Punktion mittels Seldingertechnik in der Aorta platziert, genauer, in der Aorta descendens direkt unterhalb des Abganges der linken Arteria subclavia. Direkt nach Schluss der Aortenklappe wird der Ballon mit 30-40ml Helium gefüllt, wodurch durch einen verminderten diastolischen Abfluss die Durchblutung beider Koronararterien gesteigert wird. Die IABP ist also in der Lage sowohl den linken als auch den rechten Ventrikel durch eine verbesserte Myokarddurchblutung zu unterstützen. Direkt zu Beginn der Systole wird dieser wieder leergesaugt. 
Hierdurch kommt es zu einer Senkung des Drucks in der Aorta und gleichzeitig der linksventrikulären Nachlast und Wandspannung sowie konsekutiv auch der rechtsventrikulären Nachlast und Wandspannung. Der Energiebedarf beider Ventrikel wird somit gesenkt. ${ }^{1}$

\subsubsection{Anwendung und Indikation}

Anwendung findet die IABP bei drohendem kardiogenem Schock oder therapieresistentem, mittelgradig schwerem Linksherzversagen, vor allem nach Myokardinfarkt oder nach Herzoperationen. Einer im Jahr 2016 veröffentlichten Studie zufolge, sinkt das Risiko für die Entwicklung eines Low-output-Syndroms (eingeschränkte linksventrikuläre Funktion) durch Vorwärtsversagen zumindest bei koronarchirurgischen Operationen ohne Herz-Lungen-Maschine unter Einsatz der IABP signifikant. ${ }^{16}$ Eine erfolgreiche Therapie geht so gleichzeitig mit einer niedrigeren 30-Tages-Mortalität einher. ${ }^{16} \mathrm{Zu}$ den Indikationen gehören außerdem ventrikuläre Arrhythmien, eine instabile Angina pectoris und eine schwere Mitralinsuffizienz. ${ }^{17}$ Die IABP kommt seltener auch bei Patienten mit septischem Schock zum Einsatz. Sie kann über einem Zeitraum von Tagen bis Wochen verwendet werden und die Herzfunktion unterstützen.

\subsubsection{Kontraindikationen}

Aortenaneurysmen, die schwere periphere arterielle Verschlusskrankheit (pAVK) und die höhergradige Aorteninsuffizienz, bei der es durch das zurückgepresste Blut zu irreversiblen Schäden am linken Ventrikel kommen kann, stellen Kontraindikationen für die Anwendung einer IABP dar. Bei schwerem Linksherzversagen, biventrikulärem Herzversagen oder schweren Schockzuständen gelangt die IABP ebenfalls an ihre Grenzen.

\subsubsection{Komplikationen}

Die Komplikationen im Rahmen einer IABP-Therapie lassen sich meist auf die Punktion zurückführen. So kann es distal der Punktionsstelle an der Arteria femoralis zu Ischämien oder Embolien kommen. Gleichzeitig ist die Entstehung eines Aneurysma spuriums ebenfalls möglich. Das Thromboserisiko ist ebenfalls erhöht. ${ }^{18}$ In circa einem Prozent der Fälle sind non-okklusive mesenteriale Ischämien auf eine IABP zurückzuführen. ${ }^{1}$ 


\subsection{Zielsetzung der Arbeit}

Nach Ma et al. sind ECLS und IABP einander ergänzende Therapien, die sich synergistisch bei der Behandlung von akuten Herzkrankheiten auswirken können. ${ }^{19}$ Ro et al. haben festgestellt, dass beim begleitenden Einsatz von IABP und ECLS der Weaningerfolg der behandelten Patienten signifikant größer ist als bei Patienten, die allein mit ECLS behandelt wurden. ${ }^{20}$ Ein signifikanter Unterschied bezüglich der Krankenhausmortalität konnte in dieser Studie zwischen den beiden Therapieformen jedoch nicht festgestellt werden. ${ }^{20}$ Es gibt in den letzten Jahren jedoch auch immer mehr Studien, die keinen überzeugenden Vorteil des Einsatzes der IABP bei (Infarkt-assoziiertem) kardiogenem Schock nachweisen können. ${ }^{21,22}$

Ziel dieser Arbeit war es insbesondere herauszufinden, ob nun der gleichzeitige Einsatz der IABP bei ECLS- Therapie von Vor- oder sogar von Nachteil für das Outcome der Patienten ist. Ebenfalls ist der Vergleich möglicherweise zusammenhängender Komplikationen, wie z.B. der Extremitätenischämie oder dem akuten Nierenversagen, Teil dieser Untersuchung. 


\section{Patienten und Methodik}

\subsection{Datenerhebung und Statistik}

Die Rohdaten zu den erstellten Statistiken wurden durch Einsicht in die Patientenakten des digitalen Archivs des Krankenhaus-Informationssystems Orbis $\AA^{\circledR}$ des Universitätsklinikums Frankfurt und das Kardiotechnikerprotokoll sowie durch telefonischen Kontakt mit Überlebenden bzw. Hinterbliebenen oder dem Hausarzt des jeweiligen Patienten erhoben. Die statistische Auswertung erfolgte mithilfe der Statistiksoftware BiAS (Version 11.09 - 05/18 @epsilonVerlag). Für die Vergleichbarkeit der untersuchten Patientengruppen wurde in Zusammenarbeit und unter Supervision des Instituts für Biostatistik des Universitätsklinikums Frankfurt ein Propensity Score Matching durchgeführt. Die statistische Signifikanz der Ergebnisse wurde mittels p-Wert und Standardabweichung getestet. Mit dem Kaplan-Meier-Verfahren wurden die Überlebenszeiten analysiert und die entsprechenden Konfidenzbereiche als HallWellner-Konfidenzbänder graphisch dargestellt (siehe Kapitel 3.3). Der statistische Vergleich der Überlebensraten erfolgte mithilfe des Log-Rank Tests.

\subsection{Patientenpopulation}

Im Rahmen dieser retrospektiven Studie wurden alle Patienten eingeschlossen, die in der Klinik für Herz- und Thorax - und thorakale Gefäßchirurgie der Universitätsklinik Frankfurt am Main im Zeitraum von Dezember 2001 bis Ende 2013 eine ECLS-Therapie erhalten haben. Mithilfe des Propensity Score Matchings wurden aus dem Gesamtpatientenkollektiv zwei Datensätze mit jeweils 59 Patienten gebildet. Der erste Datensatz enthält 59 Patienten, die eine reine ECLS-Therapie erhalten haben (im Folgenden IABP--Gruppe genannt), während der zweite Datensatz 59 Patienten enthält, die mit ECLS und zusätzlich einer IABP behandelt wurden (im Weiteren IABP+-Gruppe genannt). Die beiden Patientenkollektive sind hinsichtlich relevanter Risikofaktoren vergleichbar.

\subsubsection{Risikoprofil der Gesamtpopulation}

Zur Evaluation des Outcomes und zur Erstellung von passenden Matchingpartnern, wurden folgende Parameter gesammelt: Geschlecht, Alter, Gewicht, linksventrikuläre Ejektionsfraktion, Intubationsstatus bei Einlieferung, 
das Vorliegen eines chronischen Vorhofflimmerns, einer arteriellen Hypertonie, einer pulmonalen Hypertonie, eines Diabetes mellitus Typ II, einer Hyperlipoproteinämie, eines Nikotinabusus, eines NYHA (New York Heart Association)-Stadiums III oder IV, eines akuten Myokardinfarkts, einer kardiopulmonalen Reanimation (CPR) vor ECLS-Implantation und ob eine IABP zusätzlich verwendet wurde oder nicht. Die verschiedenen Stadien der Herzinsuffizienz wurden von der New York Heart Associaton folgendermaßen definiert (Tabelle 1). ${ }^{15}$

Tabelle 1: Stadieneinteilung der Herzinsuffizienz nach NYHA

\begin{tabular}{|c|c|c|}
\hline $\begin{array}{l}\text { NYHA- } \\
\text { Stadium }\end{array}$ & funktionelle Klassifizierung & objektiver Befund \\
\hline I & $\begin{array}{l}\text { Herzerkrankung ohne körperliche } \\
\text { Einschränkung bei gewohnter } \\
\text { Leistungsfähigkeit. } \\
\text { Alltägliche körperliche Belastung } \\
\text { verursacht keine inadäquate } \\
\text { Erschöpfung, Rhythmusstörungen oder } \\
\text { Angina pectoris }\end{array}$ & $\begin{array}{l}\text { keine objektiven Hinweise für } \\
\text { eine Herz-Kreislauf- } \\
\text { Erkrankung }\end{array}$ \\
\hline II & $\begin{array}{l}\text { Patienten mit Herzerkrankung und } \\
\text { leichter Einschränkung der gewohnten } \\
\text { körperlichen Leistungsfähigkeit. } \\
\text { Keine Beschwerden in Ruhe, alltägliche } \\
\text { körperliche Belastung verursacht } \\
\text { Erschöpfung, Rhythmusstörungen, } \\
\text { Luftnot oder Angina pectoris }\end{array}$ & $\begin{array}{l}\text { objektive Hinweise für } \\
\text { minimale Herz-Kreislauf- } \\
\text { Erkrankung }\end{array}$ \\
\hline III & $\begin{array}{l}\text { Patienten mit Herzerkrankung und } \\
\text { höhergradiger Einschränkung der } \\
\text { gewohnten körperlichen } \\
\text { Leistungsfähigkeit. } \\
\text { Keine Beschwerden in Ruhe, geringe } \\
\text { körperliche Belastung verursacht } \\
\text { Erschöpfung, Rhythmusstörungen, } \\
\text { Luftnot oder Angina pectoris }\end{array}$ & $\begin{array}{l}\text { objektive Hinweise für mäßig } \\
\text { bis höhergradige Herz- } \\
\text { Kreislauf-Erkrankung }\end{array}$ \\
\hline IV & $\begin{array}{l}\text { Patienten mit Herzerkrankungen. } \\
\text { Beschwerden bei allen körperlichen } \\
\text { Aktivitäten und teilweise in Ruhe }\end{array}$ & $\begin{array}{l}\text { objektive Hinweise für schwere } \\
\text { Herz-Kreislauf-Erkrankung }\end{array}$ \\
\hline
\end{tabular}


Darüber hinaus wurden die Daten der individuell aufgetretenen Komplikationen erhoben. Dazu gehören Blutungen an der Kanülierungsstelle, Extremitätenischämien, Nierenversagen, die Notwendigkeit einer Rethorakotomie, das Auftreten eines Kompartmentsyndroms oder einer Infektion sowie neurologische Komplikationen wie Schlaganfälle oder Hirnblutungen.

Die Abbildung 5 zeigt die Verteilung der aufgeführten Risikofaktoren innerhalb der beiden Patientenkollektive.

\section{Kardiovaskuläre Risikofaktoren}

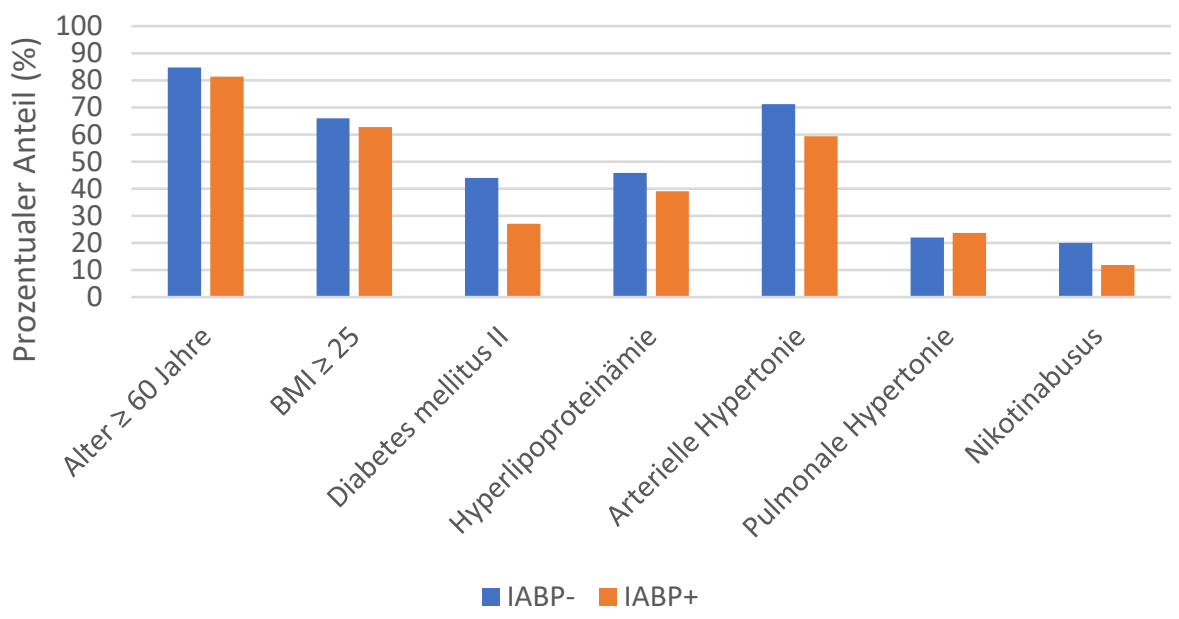

Abbildung 5: Kardiovaskuläre Risikofaktoren der beiden Patientenkollektive

\subsubsection{Rahmenbedingungen der Implantation}

Durch die unterschiedlichen Indikationen für eine ECLS-Implantation kommen auch gleichzeitig unterschiedliche Rahmenbedingungen zustande. So entsteht der Parameter der kardiopulmonalen Reanimation, welche in besonders schweren Fällen angewandt werden musste. In anderen Fällen wurde, um eine verbesserte Perfusion der Koronararterien sowie eine Senkung der Nachlast zu erreichen, zusätzlich oder vor Implantation eines ECLS-Systems auch eine Intraaortale Ballonpumpe (IABP) implantiert. 


\subsubsection{Operationen des Gesamtkollektivs}

Die in dieser Studie untersuchten Patienten erhielten je nach Ursache für das kardiopulmonale Versagen unterschiedliche Operationen. Zu diesen gehören herzchirurgische Eingriffe wie Koronararterienbypässe, Koronararterienbypässe in Kombination mit Klappenrekonstruktionen, alleinige Klappenrekonstruktionen, Eingriffe an der Aorta sowie das Einsetzen eines Herzunterstützungssystems (LVAD, Left Ventricular Assist Device) mit und ohne gleichzeitige Klappenrekonstruktion. Zu den im Patientenkollektiv seltener stattgehabten Operationen gehören eine Herz- oder Lungentransplantation, die Korrektur eines Ventrikelseptumdefekts, eine Perikardektomie und eine tracheale Rekonstruktion.

\subsection{Monitoring unter laufender Extracorporeal Life Support -Therapie}

Da es sich bei Patienten mit Bedarf einer ECLS-Therapie um Hochrisikopatienten handelt, die sich in Lebensgefahr befinden, ist es unerlässlich regelmäßige Blutuntersuchungen durchzuführen, um später prognostische Aussagen treffen zu können. Hierbei wird zwischen konventionellen Laboruntersuchungen und Blut-Gas-Analysen unterschieden.

\subsection{Procedere auf der Intensivstation}

Sobald die Patienten postoperativ auf die Intensivstation aufgenommen werden, kommt ihnen ein streng kontrolliertes Patientenmanagement zuteil. Hierzu gehört eine regelmäßige, ausführliche körperliche Untersuchung (zweimal täglich). Schwerpunkt dieser Untersuchung liegt bei der frühzeitigen Erkennung von Ischämiezeichen und Blutungen. Hierzu werden vor allem Lunge, Abdomen, Extremitäten und die Neurologie überprüft. Durch die Anfertigung eines Röntgenbildes wird täglich die optimale Lage der Kanülen und der IABP geprüft. Um die ECLS - Therapie zu optimieren wird in regelmäßigen Abständen eine transösophageale Echokardiographie (TEE) durchgeführt, durch die die myokardiale Kontraktilität und die Ventrikelfüllung evaluiert wird. Begleitend kann das Vorliegen eines Perikardergusses ausgeschlossen werden kann. Das ECLSSystem selbst wird zweimal pro Tag von einem Kardiotechniker auf volle Funktionstüchtigkeit überprüft. Er sucht nach Thromben oder Kontaktstörungen und prüft den Oxygenator sowie das Schlauchsystem. 
Alle 2-4 Stunden wird außerdem eine Blutgasanalyse zur Erfassung des klinischen Status und der Patientenmetabolik durchgeführt. Zur vierstündlichen Blutuntersuchung gehört die Abnahme von Gerinnungswerten (Quick, aPTT) und Herzenzymen (CK, CK-MB, Troponin). Leber- und Nierenwerte werden zwölfstündlich überprüft. Außerdem ist auch die Durchführung einer Rotationsspektrometrie (@ Multiplate) möglich, durch welche bei Bedarf sofort mit den entsprechenden Blutprodukten einem Gerinnungsproblem (wie zum Beispiel einer Thrombozyten- funktionsstörung) entgegengearbeitet werden kann.

\subsubsection{Beatmung}

Die invasive Beatmung der Intensivpatienten erfolgt immer so lungenschonend und minimalinvasiv wie möglich über einen Endotrachealtubus oder, wie es bei Langzeitbeatmung der Fall wäre, ein Tracheostoma. Man versucht einen möglichst niedrigen endinspiratorischen und einen erhöhten positivendexspiratorischen Druck zu erreichen, um ein bedarfsadaptiertes Tidalvolumen zu erzielen.

\subsubsection{Medikation}

Alle Patienten erhalten eine Sedierung mittels einer Kombination aus Opiat und Benzodiazepin oder Alpha-2-Agonisten (Clonidin/ Dexmedetomidin). Bei Bedarf wird auch Propofol eingesetzt, allerdings aufgrund der Nebenwirkung der Kreislaufdepression nur bei kreislaufstabilen Patienten mit niedrigem Vasopressorbedarf.

Alle Patienten, bei denen eine Myokardrevaskularisation durchgeführt wurde, erhalten Aspirin. Außerdem werden die Patienten mit Katecholaminen und analgetischen Präparaten, Opiaten, je nach Bedarf versorgt. Eine parenterale Ernährung sowie eine empirische antibiotische Therapie mit Vancomycin und einem Cephalosporin (i.d.R. Cefuroxim) für die ersten 48 Stunden gehören ebenfalls zum vorgegebenen Standard bei Patienten mit ECLS-Therapie. Danach wird eine kalkulierte oder eine durch ein Antibiogramm angepasste Antibiotikatherapie durchgeführt. 


\subsubsection{Entwöhnung von der Extracorporeal Life Support - Therapie (Weaning)}

Da die ECLS - Therapie keinesfalls als Dauertherapie gesehen werden darf, sondern primär dazu dient, den betroffenen Organsystemen Erholungszeit zu gewährleisten, muss möglichst früh an eine schrittweise Entwöhnung (Weaning) gedacht werden. Diese wird optimalerweise nach 24 bis 72 Stunden maximalen ECLS-Flusses initiiert.

Beim Weaning wird der Fluss um 0,5 $\mathrm{l} / \mathrm{min} /$ Tag reduziert, sofern der Patient auch unter der Reduktion hämodynamisch und metabolisch stabil bleibt. Die hämodynamische Kontrolle erfolgt üblicherweise mittels transösophagealer Echokardiographie. Ziel ist es, eine ausreichende Funktion beider Ventrikel zu gewährleisten oder gegebenenfalls die Ventrikelfunktion durch Einsatz von Inotropika (Suprarenin, Dobutamin, etc.) zu verbessern. Die zentralvenöse Sättigung sollte nicht unter $70 \%$ fallen und der Sauerstoffpartialdruck sollte mindestens zwischen 70 und $80 \mathrm{mmHg}$ liegen. Sollte dies nicht der Fall sein, wird der ECLS-Fluss wieder erhöht. Die Flussreduktion wird bei $2 \mathrm{l} / \mathrm{min}$ gestoppt, da sonst die Gefahr für eine Gerinnselbildung im System deutlich erhöht ist. 


\section{Ergebnisse}

\subsection{Demographische Daten}

Das der Studie zugrunde liegende Patientenkollektiv besteht aus insgesamt 118 Patienten (15 Frauen, 103 Männer). 59 Patienten (7 Frauen, 52 Männer) wurden nach kardiochirurgischen Eingriffen bzw. bei respiratorischer Insuffizienz mittels ECLS mechanisch unterstützt (IABP'-Gruppe). Bei weiteren 59 Patienten (8 Frauen, 51 Männer) erfolgte die ECLS - Unterstützung in Kombination mit dem zusätzlichen, gleichzeitigen Einsatz von IABP (IABP+-Gruppe).

Die beiden Patientenkollektive wurden mithilfe eines Propensity Score Matchings aus allen im Zeitraum zwischen Dezember 2001 bis Ende 2013 behandelten Patienten ausgewählt.

In Tabelle 2 sind wesentliche Daten der durchgeführten Studie für die beiden Patientenkollektive zusammenfassend dargestellt.

Tabelle 2: Demographik

\begin{tabular}{|l|l|l|}
\hline & IABP-Gruppe & IABP+-Gruppe \\
\hline Patientenzahl (n) & 59 & 59 \\
\hline Durchschnittsalter (Jahre) & $68,7+/-10$ & $67,8+/-11,5$ \\
\hline $\begin{array}{l}\text { Durchschnittliche Unterstützungszeit } \\
\text { (Tage) }\end{array}$ & $7,9+/-9,5$ & $6,4+/-4,3$ \\
\hline $\begin{array}{l}\text { Durchschnittliche Dauer des } \\
\text { Krankenhausaufenthalts (Tage) }\end{array}$ & $22,4+/-22,4$ & $21,5+/-28,5$ \\
\hline $\begin{array}{l}\text { ICU-Stay (mittlere Verweildauer auf der } \\
\text { Intensivstation in Tagen) }\end{array}$ & $17,9+/-22,0$ & $17,1+/-24,1$ \\
\hline Durchschnittsalter Überlebende (Jahre) & $65,5+/-11,9$ & $65,4+/-7,5$ \\
\hline
\end{tabular}


Der Altersdurchschnitt beträgt 68,7 +/- 10 Jahre in der IABP--Gruppe und 67,8 +/- 11,5 Jahre in der IABP+-Gruppe. Den Abbildungen 6 und 7 ist für die beiden Patientenkollektive die in Altersgruppen von jeweils zehn Jahren unterteilte Alters- und Geschlechtsverteilung zu entnehmen.

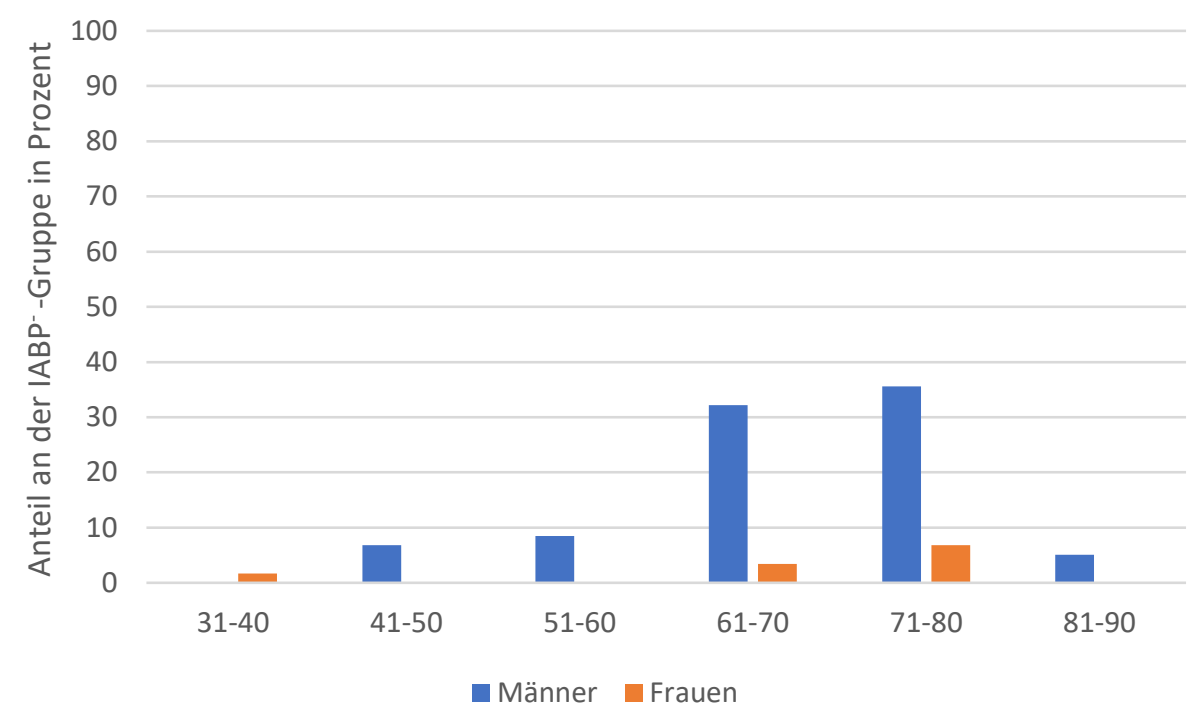

Abbildung 6: Alters- und Geschlechtsverteilung in der IABP--Gruppe ( $n=59)$

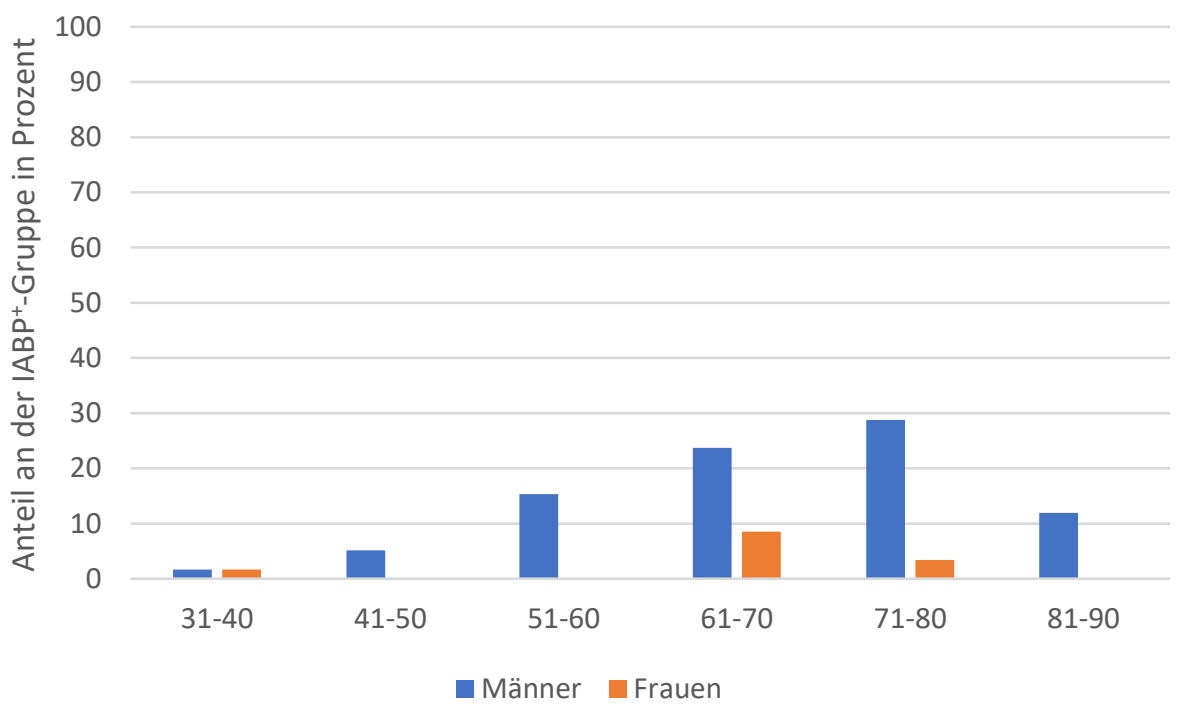

Abbildung 7: Alters- und Geschlechtsverteilung in der IABP+-Gruppe ( $n=59)$ 


\subsection{Operative Eingriffe}

Die in dieser Studie untersuchten Patienten erhielten unterschiedliche Operationen (Abbildung 8). Die Verteilung der Operationen in den beiden Vergleichsgruppen (IABP+ ${ }^{+}$und IABP-) ist der Abbildung $9 \mathrm{zu}$ entnehmen. $\mathrm{Zu}$ diesen gehört je nach Notwendigkeit in $61 \%$ der Fälle die Anlage eines Koronararterienbypasses (Aorto-Coronarer-Venen-Bypass, ACVB), in $12 \%$ der Fälle die Korrektur einer Herzklappe, sowie in $23 \%$ die Kombination aus beidem. Unter die übrigen 12\% der Prozeduren (Sonstige) gehören eine Lungen- oder Herztransplantation, eine tracheale Rekonstruktion, die Korrektur eines Ventrikelseptumdefekts oder eine Perikardektomie. Die Aufteilung der Operationen in den beiden Gruppen IABP+ und IABP- unterscheidet sich um maximal drei Prozentpunkte.

\section{Gesamtpatientenkollektiv}

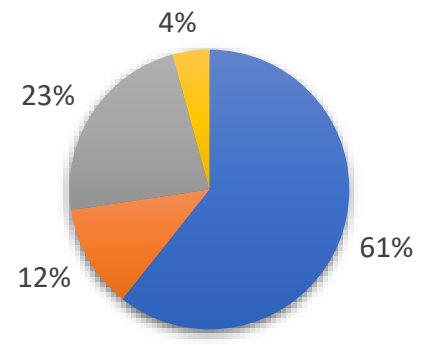

- ACVB allein $\square$ Klappenkorrektur $\square$ ACVB mit Klappenkorrektur $\square$ Sonstige

\section{Abbildung 8: Operative Eingriffe am Gesamtkollektiv}

\section{IABP-}

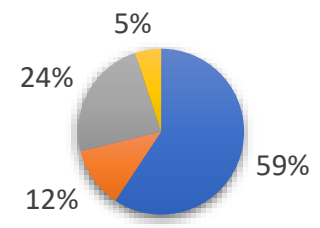

- ACVB allein

- Klappenkorrektur

aCVB mit Klappenkorrektur

Sonstige

\section{$\mathrm{IABP}^{+}$}

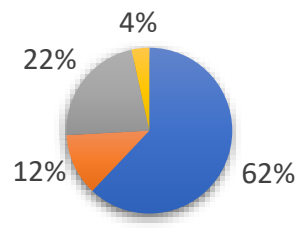

- ACVB allein

Klappenkorrektur

aCVB mit Klappenkorrektur

- Sonstige

Abbildung 9: Operative Eingriffe in der IABP-- und IABP+- Gruppe 


\subsection{Mortalität}

In der IABP-Gruppe konnten von der Gesamtpatientenzahl $(n=59)$ insgesamt 24 Patienten (40,7\%) erfolgreich von der ECLS-Unterstützung entwöhnt werden. Jedoch verstarben 14 Patienten $(23,7 \%)$ nach erfolgreicher Explantation. Dies ergibt eine Gesamtmortalität von $83 \%$.

In der IABP+-Gruppe konnten von der Gesamtpatientenzahl $(n=59)$ insgesamt 22 Patienten (37,3\%) erfolgreich von der ECLS/IABP-Unterstützung entwöhnt werden. Jedoch verstarben 10 Patienten (17\%) nach erfolgreicher Explantation. Dies ergibt eine Gesamtmortalität von 79,7\%.

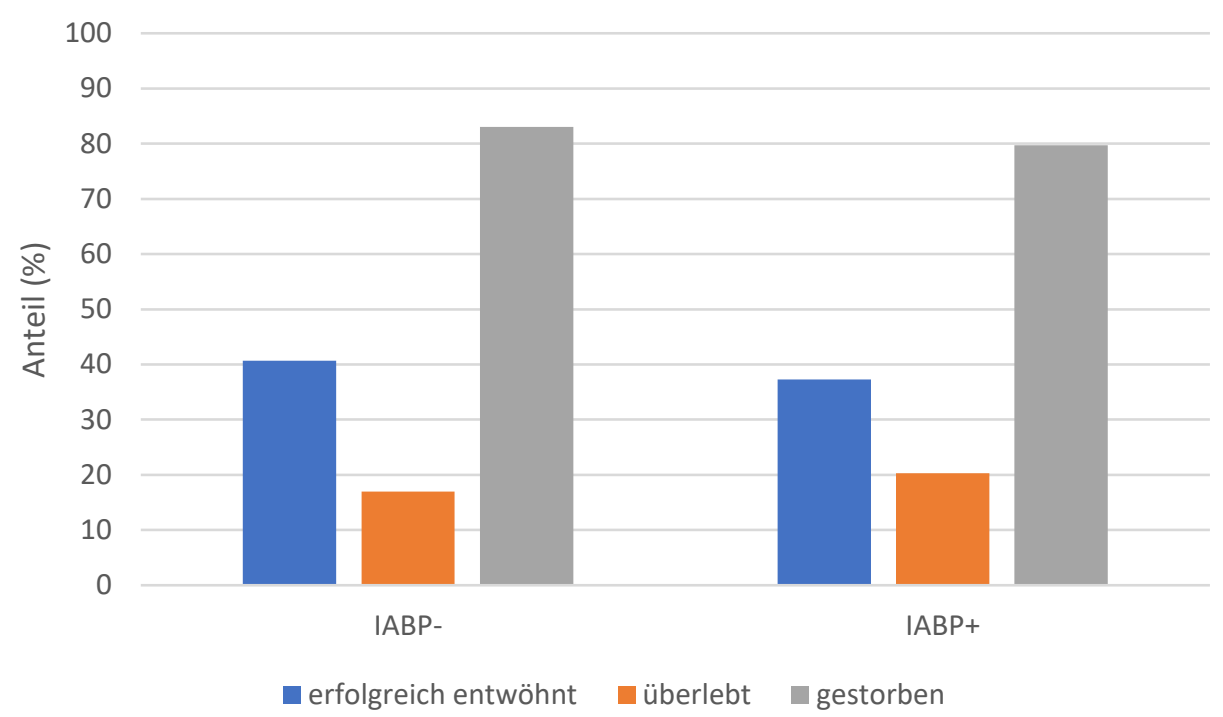

Abbildung 10: Prozentuale Anteile der erfolgreich entwöhnten, verstorbenen und überlebenden Patienten

In Abbildung 10 ist für die beiden Patientenkollektive jeweils der prozentuale Anteil der erfolgreich entwöhnten, verstorbenen und überlebenden Patienten graphisch dargestellt.

Die Überlebenszeiten in den beiden Patientenkollektiven wurden mit dem Kaplan-Meier-Verfahren analysiert (BiAS Version 11.09 - 05/18 CepsilonVerlag). Die mit diesem Verfahren ermittelten Überlebensraten geben an, wie viele der behandelten Patienten bis zu einem bestimmten Zeitpunkt überleben. Danach beträgt das strukturelle Überleben im Hinblick auf 30 Tage $33 \%$ für die IABP--Gruppe und 25,1 \% für die IABP+-Gruppe. Der Verlauf der Überlebensrate für die beiden Patientengruppen für den Zeitraum von 30 Tagen ist den 
Abbildungen 11, 12 und 13 zu entnehmen. Für das Gesamtpatientenkollektiv beträgt das strukturelle Überleben im Hinblick auf 30 Tage 30 \% (Abbildung 14).

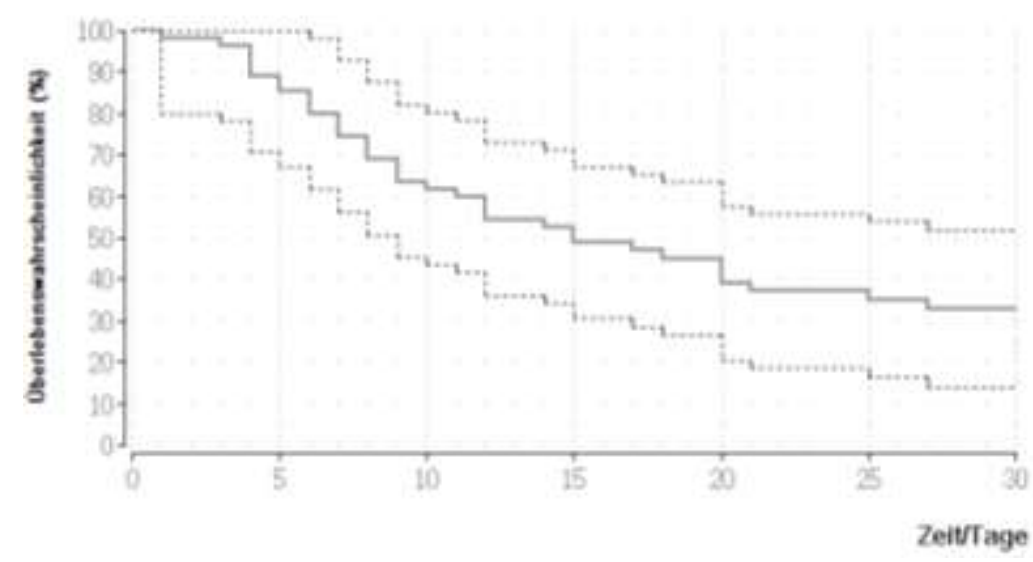

Abbildung 11: 30-Tages-Überlebensrate mit Konfidenzbereich $P=0,95$ für die IABP--Gruppe (n = 59)

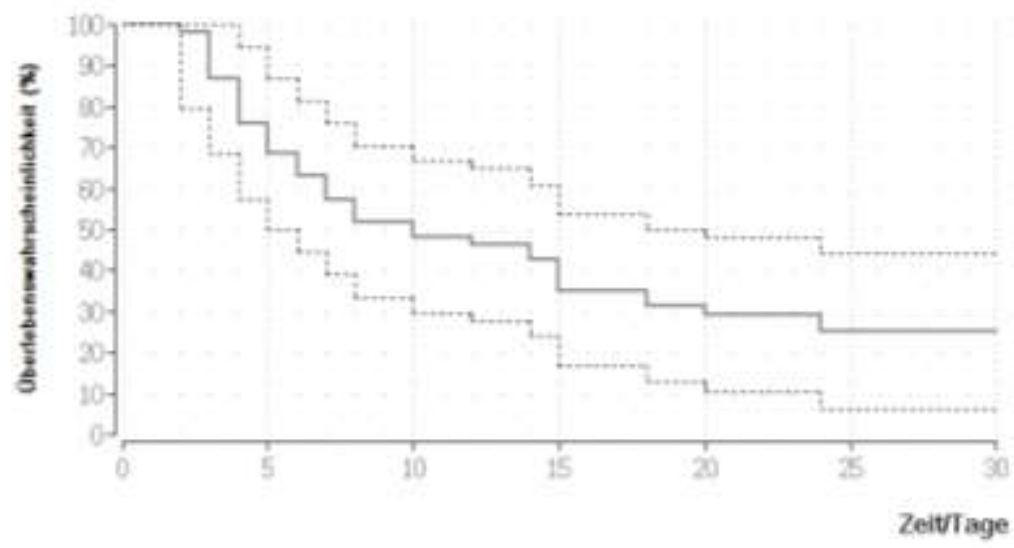

Abbildung 12: 30-Tages-Überlebensrate mit Konfidenzbereich $P=0,95$ für die IABP+-Gruppe ( $n=59)$

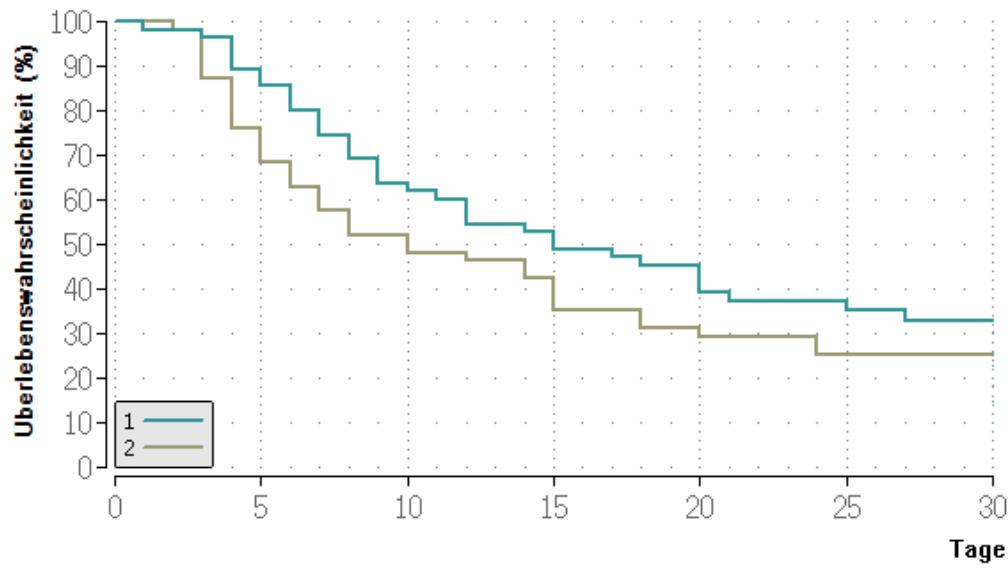

Abbildung 13: 30-Tages-Überlebensrate (1: IABP+-Gruppe, 2: IABP--Gruppe) 


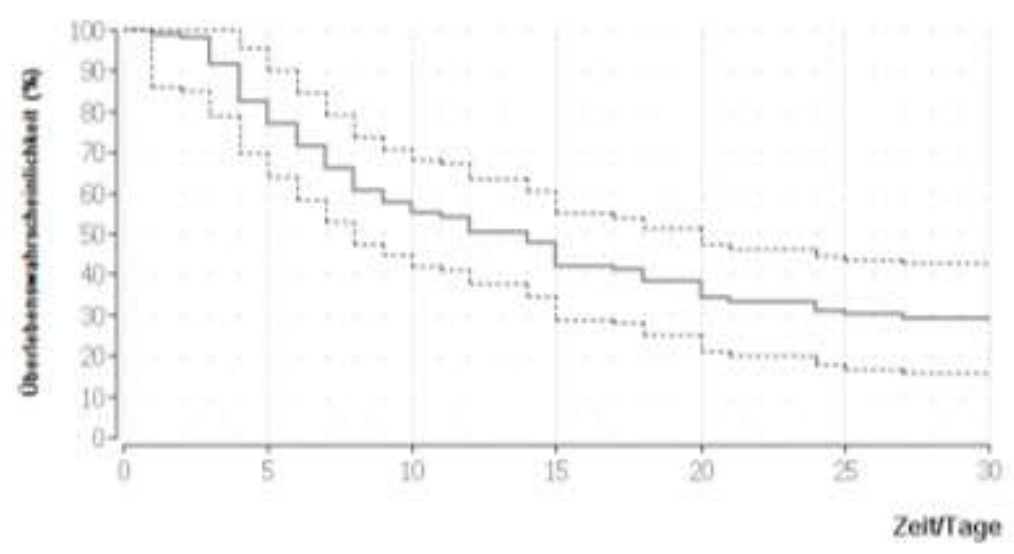

Abbildung 14: 30-Tages-Überlebensrate mit Konfidenzbereich $P=0,95$ für das Gesamtkollektiv $(n=118)$

\subsection{Todesursachen}

Von den in dieser Studie insgesamt mit ECLS behandelten 118 Patienten verstarben 96 Patienten noch in der Klinik. In der IABP--Gruppe verstarben 49 Patienten, in der $\mathrm{IABP}^{+}-$Gruppe waren es 47 Patienten. Im Einzelnen sind folgende Todesursachen aufgetreten:

- therapierefraktäres Multiorganversagen (43\%)

- persistierender kardiogener Schock (20\%)

- Sepsis (13\%)

- hypovolämischer Schock (2\%)

- infauste neurologische Prognose (8\%)

- sonstige Ursachen (14\%)

Abbildung 15 und 16 zeigen die prozentuale Verteilung der Haupttodesursachen an der Gesamtmortalität für die beiden Patientengruppen. Die Todesursachen des Gesamtkollektivs sind der Abbildung 17 zu entnehmen. 


\section{IABP--Gruppe}

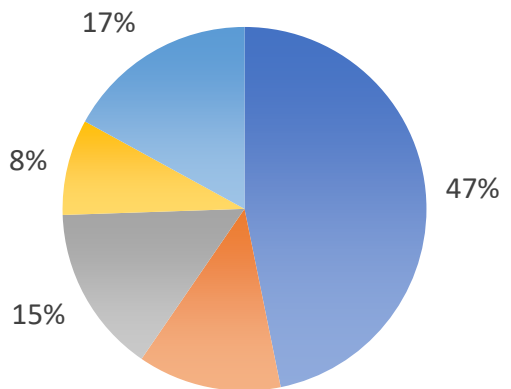

$13 \%$
Multiorganversagen

kardiogener Schock

Sepsis

infauste neurologische Prognose

Sonstige

\section{Abbildung 15: Todesursachen in der IABP'-Gruppe}

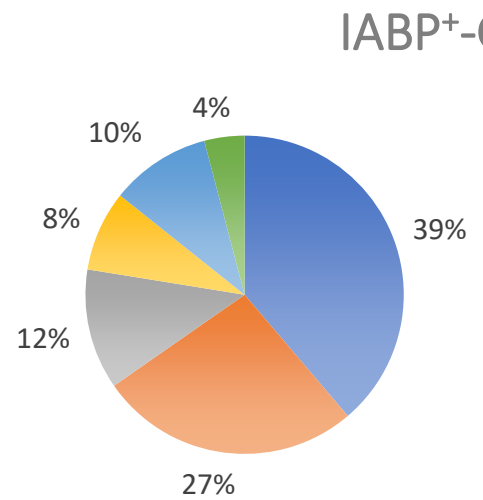
Multiorganversagen
kardiogener Schock
- Sepsis
infauste neurologische Prognose
Sonstige
hypovolämischer Schock

\section{Abbildung 16: Todesursachen in der IABP+-Gruppe}

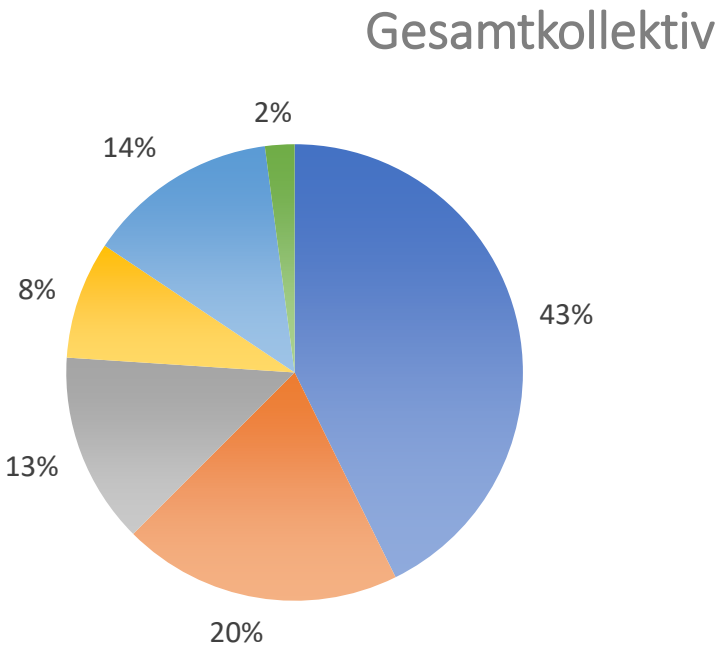

- Multiorganversagen

kardiogener Schock

Sepsis

infauste neurologische Prognose

- Sonstige

hypovolämischer Schock

\section{Abbildung 17: Todesursachen des Gesamtkollektivs}




\subsection{Komplikationen}

Die Abbildungen 18 und 19 zeigen den prozentualen Anteil der unter ECLSTherapie aufgetretenen Komplikationen in beiden Patientengruppen. Zu diesen gehören eine Rethorakotomie, eine Blutung an der Kanülierungsstelle, eine Infektion, eine Extremitätenischämie, Nierenversagen, aber auch neurologische Komplikationen, die unter der Therapie aufgetreten sind.



Abbildung 18: Aufgetretene Komplikation ohne Einsatz einer IABP

IABP + -Gruppe



Abbildung 19: Aufgetretene Komplikation bei zusätzlichem Einsatz einer IABP 


\subsubsection{Rethorakotomie}

Im Folgenden wurde analysiert welche Patienten im Rahmen der initialen Operation oder nach Implantation des ECLS-/ECLS+IABP - Systems aufgrund von Blutung oder Herzbeuteltamponade erneut chirurgisch revidiert werden mussten. Insgesamt mussten 66 Patienten rethorakotomiert werden (55,9\%). In der IABP+-Gruppe waren es 36 Patienten (61\%). In der IABP--Gruppe mussten 30 Patienten $(50,8 \%)$ aufgrund einer Herzbeuteltamponade oder einer manifesten intrathorakalen Blutung chirurgisch revidiert werden. Dies ergibt eine Odds-Ratio (Chancenverhältnis) für die Gesamtpopulation von 1,64. Das Signifikanzlevel liegt bei $P=0.28$.

\subsubsection{Blutung an der Kanülierungsstelle}

Der nächste Parameter widmet sich der Frage, ob im Rahmen der ECLSBehandlung im Bereich der arteriellen Kanülenanlage (A. subclavia rechts, Aa. femorales) eine chirurgische revisionspflichtige Blutung aufgetreten ist. Von den insgesamt 118 untersuchten Patienten zeigten 29 Patienten (24,6\%) eine Blutung an der Kanülierungsstelle (IABP+-Gruppe: 18 Patienten (30,5\%); IABP-Gruppe: 11 Patienten (18,6\%). Hierfür zeigt sich eine Gesamt-Odds-Ratio von 1,41. Das Signifikanzlevel liegt bei $P=0,53$.

\subsubsection{Neurologische Komplikationen}

Zu den neurologischen Komplikationen zählen sowohl apoplektische Insulte mit Funktionseinschränkungen als auch intrakranielle Blutungen. Insgesamt zeigten 17 Patienten (14,4\%) eine solche neurologische Komplikation im Rahmen ihres stationären Aufenthaltes (IABP+-Gruppe: 10 Patienten (16,9\%); IABP-Gruppe: 7 Patienten (11,9\%)). Die Odds-Ratio liegt bei 2. Das statistische Signifikanzlevel liegt bei $P=0,35$. 


\subsubsection{Extremitätenischämie}

Mit diesem Parameter wurden alle beobachteten Extremitätenischämien (Verschluss, notwendige Gefäßchirurgische Revision, Amputation, Nekrose) analysiert. Insgesamt zeigten 17 Patienten (14,4\%) eine solche Extremitätenischämie (IABP+-Gruppe: 9 Patienten (15,3\%); IABP-Gruppe: 8 Patienten $(13,6 \%))$.

Dies ergibt eine Odds-Ratio von 4,72. Das statistische Signifikanzlevel liegt bei $P=0,14$.

\subsubsection{Infektion/Sepsis}

Unter diesem Punkt wurden alle Patienten aufgeführt, bei denen im Rahmen des stationären Aufenthaltes eine therapiebedürftige Infektion/ Sepsis auftrat. Insgesamt wiesen 40 (33,9\%) Patienten eine solche Infektion/ Sepsis auf (IABP+Gruppe: 21 Patienten (35,6\%), IABP--Gruppe 19 Patienten (32,2\%)). Dies ergibt eine Odds-Ratio von 2,25. Das statistische Signifikanzlevel liegt bei $P=0,14$.

\subsubsection{Akutes Nierenversagen}

Dieser Parameter erfasst alle Patienten, welche im Rahmen der ECLS-Therapie ein akutes Nierenversagen (ANV) erwarben und zum Teil sogar eine Nierenersatztherapie (Dialyse) benötigten. Die Klassifizierung erfolgte anhand der RIFLE- oder AKIN-Kriterien. ${ }^{23,24}$ In beiden Gruppen zeigten insgesamt 91 Patienten (76\%) ein ANV (IABP+-Gruppe: 43 Patienten (72,9\%), IABP--Gruppe: 48 Patienten $(81,4 \%))$. Dies ergibt eine Odds-Ratio von 1,97. Das statistische Signifikanzlevel liegt bei $P=0,18$. 


\section{Diskussion}

59 Patienten wurden nach kardiochirurgischen Eingriffen bzw. respiratorischer Insuffizienz mit Hilfe von ECLS behandelt (IABP-Gruppe). Bei weiteren 59 Patienten wurde ECLS in Kombination mit IABP angewandt (IABP+-Gruppe).

Die Überlebenszeiten der beiden Patientenkollektive wurden mit Kaplan-MeierKurven (BiAS Version 11.09 - 05/18 @epsilon-Verlag) analysiert. Der statistische Vergleich der Überlebensraten der beiden Patientenkollektive erfolgte mit Hilfe des Log-rank-Tests (BiAS Version 11.09 - 05/18 @epsilon-Verlag). Mit diesem Testverfahren wird überprüft, ob sich die Überlebenszeiten der beiden Therapiegruppen mit oder ohne IABP signifikant unterscheiden.

\subsection{Mortalität}

Die Auswertung der erhobenen Daten hat ergeben, dass bei der kombinierten Anwendung von ECLS und IABP im Vergleich zur ECLS-Behandlung ohne IABP kein signifikanter Unterschied bei der 30-Tages-Überlebensrate der Patienten (17\% in der IABP--Gruppe und $20,3 \%$ in der IABP+-Gruppe) besteht $\left(\mathrm{Chi}^{2}=0,303, \mathrm{P}=0,582\right.$, Signifikanzniveau Alpha=0,05).

Zur gleichen Aussage kommen Park et al. , die ebenfalls festgestellt haben, dass die kombinierte Anwendung von ECLS und IABP zu keiner geringeren Krankenhausmortalität geführt hat. ${ }^{25}$

In einer breit angelegten Studie haben Lin et al. die Patientendaten von insgesamt 529 Patienten, die nach einem kardiogenen Schock mit ECLS bzw. mit ECLS in Kombination mit IABP therapiert wurden, analysiert. 227 Patienten wurden mit ECLS und 302 Patienten mit ECLS in Kombination mit IABP unterstützt. Lin et al. haben festgestellt, dass sich die 14-Tages-Mortalitätsraten in den beiden Patientengruppen nicht signifikant unterscheiden $(48,5 \%$ in der IABP--Gruppe, 47,7\% in der IABP+-Gruppe). Als Haupttodesursache wird, wie auch in der vorliegen Studie, das Multiorganversagen genannt $(92,2 \%$ in der IABP--Gruppe, 94,2\% in der (ABP+-Gruppe). ${ }^{22}$

Nach Aso et al. ist bei Patienten mit kardiogenem Schock die 28-Tages- und Krankenhausmortalität in der IABP+-Gruppe signifikant kleiner als in der IABP-Gruppe ( $48,4 \%$ vs. $58,2 \%$; $P=0,001$ und $55,9 \%$ vs. $64,5 \%$; $P=0,004) .{ }^{26} \mathrm{Li}$ et al. kommen bei einer Auswertung von 29 Studien mit insgesamt 4576 Patienten zu 
dem Ergebnis, dass die Krankenhausmortalität in der IABP+-Gruppe (58,4\%) signifikant kleiner ist als in der IABP--Gruppe $(63,1 \%) .{ }^{27}$ Ein möglicher Grund könnte die Neutralisierung unerwünschter Effekte der reinen ECLS-Therapie, wie beispielsweise die Reduktion der Nachlasterhöhung der ECLS-Therapie durch die zusätzliche IABP-Implantation oder die bessere Koronarperfusion bei erhöhtem myokardialen Sauerstoffbedarf durch die ECLS-Therapie, sein. ${ }^{28}$ Madershahian et al. haben außerdem gezeigt, dass durch den zusätzlichen Einsatz einer IABP der Flow in Koronararterienbypässen erhöht werden konnte. ${ }^{29}$ In einer anderen Studie aus dem Jahr 2007 fand man heraus, dass die Sauerstoffversorgung des Herzmuskels durch den zusätzlichen Einsatz einer IABP verbessert werden konnte und dieser deshalb bei der Verwendung von ELCS als "bridge to recovery" in Erwägung zu ziehen ist. ${ }^{30}$

Nach Ma et al. sind ECLS und IABP einander ergänzende Methoden, die sich synergistisch auf den Behandlungserfolg auswirken können. ${ }^{19}$ In der Literatur werden sehr unterschiedliche Überlebensraten für mit ECLS behandelte Patienten angegeben. Tabelle 3 zeigt zusammenfassend die Ergebnisse einzelner ECLS-Studien. Angegeben sind Patientenzahl, Durchschnittsalter und die 30-Tage- Überlebensrate. Im Vergleich dazu ist die 30-Tage-Überlebensrate der beiden Patientengruppen dieser Studie aufgeführt. 
Tabelle 3: Vergleich der Überlebensraten mit anderen ECLS-Studien

\begin{tabular}{|l|l|l|l|}
\hline Referenz & $\begin{array}{l}\text { Patienten- } \\
\text { zahl (n) }\end{array}$ & $\begin{array}{l}\text { Durchschnitts- } \\
\text { alter (Jahre) }\end{array}$ & $\begin{array}{l}\text { 30- } \\
\text { Tage - Überleben } \\
\text { (\%) }\end{array}$ \\
\hline Muehrcke et al., 1996 & 23 & $47,3 \pm 16,4$ & 30 \\
\hline Noon et al.,1999 & 141 & 61,6 & 22 \\
\hline Magovern et al., 1999 & 55 & $62 \pm 1,4$ & 36 \\
\hline Fiser et al., 2001 & 51 & $61 \pm 1,7$ & 24 \\
\hline Smedira et al., 2001 & 202 & $55 \pm 14$ & 38 \\
\hline Ko et al., 2002 & 76 & $56,8 \pm 15,9$ & 38 \\
\hline Doll et al., 2004 & 219 & $61,3 \pm 12,1$ & 24 \\
\hline Klotz et al., 2007 & 150 & $65,9 \pm 11,7$ & 25 \\
\hline Combes et al., 2008 & 81 & $46 \pm 16$ & 42 \\
\hline Bakthiary et al., 2008 & 45 & $60,1 \pm 13,6$ & 47 \\
\hline $\begin{array}{l}\text { Papadopoulos et al., } \\
\text { 2016 }\end{array}$ & 200 & $60 \pm 17$ & 37,4 \\
\hline Vorliegende Studie & 118 & $68,2 \pm 10,7$ & 29,1 \\
\hline
\end{tabular}

In Tabelle 4 sind die Überlebensraten verschiedener Studien für die IABP-Gruppe und die $\mathrm{IABP}^{+-}$-Gruppe angegeben. Auch hier sind die ermittelten Überlebensraten der einzelnen Studien sehr unterschiedlich. Weiterhin zeigen die Werte in Tabelle 4, dass ein eindeutiger Zusammenhang zwischen Behandlungsmethode "mit IABP" und „ohne IABP" und der Überlebensrate nicht abzuleiten ist. 
Tabelle 4: Behandlungsmethode und Überlebensraten anderer Studien im Vergleich

\begin{tabular}{|c|c|c|c|c|}
\hline & $\begin{array}{l}\text { Behandlungs- } \\
\text { methode }\end{array}$ & $\begin{array}{l}\text { Patien- } \\
\text { tenzahl }\end{array}$ & $\begin{array}{l}\text { Durchschnitts- } \\
\text { alter }\end{array}$ & $\begin{array}{l}\text { 30-Tages- } \\
\text { Überleben }\end{array}$ \\
\hline $\begin{array}{l}\text { Vorliegende } \\
\text { Studie }\end{array}$ & $\begin{array}{l}\mathrm{IABP}^{-} \\
\mathrm{IABP}^{+}\end{array}$ & $\begin{array}{l}59 \\
59\end{array}$ & $\begin{array}{l}68,7+/-10 \\
67,8+/-11,5\end{array}$ & $\begin{array}{l}33 \% \\
25,1 \%\end{array}$ \\
\hline $\begin{array}{l}\text { Park, Yang } \\
\text { et. Al, } 2014\end{array}$ & $\begin{array}{l}\mathrm{IABP}^{-} \\
\mathrm{IABP}^{+}\end{array}$ & $\begin{array}{l}55 \\
41\end{array}$ & $\begin{array}{l}64(54-75) \\
66(57-74)\end{array}$ & $\begin{array}{l}53,1 \% \\
53,9 \%\end{array}$ \\
\hline $\begin{array}{l}\text { Nuding et } \\
\text { al., } 2017\end{array}$ & $\begin{array}{l}\mathrm{IABP}^{-} \\
\mathrm{IABP}^{+}\end{array}$ & $\begin{array}{l}533 \\
533\end{array}$ & $\begin{array}{l}\text { Nicht } \\
\text { angegeben }\end{array}$ & $\begin{array}{l}41,8 \%(28 d) \\
51,6 \%(28 d)\end{array}$ \\
\hline Ro et al. & $\begin{array}{l}\mathrm{IABP}^{-} \\
\mathrm{IABP}^{+}\end{array}$ & $\begin{array}{l}193 \\
60\end{array}$ & $\begin{array}{l}58,8+/-15,3 \\
\text { (gesamt) }\end{array}$ & $\begin{array}{l}28 \% \\
31,4 \% \\
\text { (Krankenhaus- } \\
\text { mortalität) }\end{array}$ \\
\hline
\end{tabular}

\subsection{Weaning}

Auch beim Weaningerfolg ist in der vorliegenden Studie kein signifikanter Unterschied zwischen den beiden Patientenkollektiven $(40,7 \%$ in der IABP-Gruppe und 37,3\% in der IABP+-Gruppe) zu verzeichnen (Chi2=0,087; $P=0,768$, Signifikanzniveau Alpha $=0,05$ ). Eine Studie von Ro et al. hat jedoch ergeben, dass beim begleitenden Einsatz von IABP und ECLS der Weaningerfolg signifikant größer ist als bei Patienten, die allein mit ECLS behandelt wurden. Aso et al. kommen ebenfalls zu dem Ergebnis, dass der Weaningerfolg in der IABP+Gruppe -Gruppe signifikant größer ist ( $82,6 \%$ vs. $73,4 \%$; $P<0,001) .{ }^{26}$

Einen signifikanten Unterschied bei der Krankenhausmortalität haben die Autoren jedoch nicht festgestellt. ${ }^{20}$

\subsection{Risikofaktoren}

Abbildung 20 zeigt für diese Studie die Altersverteilung der erfolgreich behandelten Patienten für das Gesamtpatientenkollektiv ( $n=118$ ). Die erfolgreich behandelten Patienten haben ein Alter zwischen 46 und 78 Jahren. Das durchschnittliche Alter der erfolgreich behandelten Patienten liegt bei $65,5+/-9,5$ Jahren. 
Im Vergleich dazu beträgt das durchschnittliche Alter aller behandelten Patienten 68,2 +/-10,7 Jahre. Somit spricht ein höheres Patientenalter für ein schlechteres Outcome unter ECLS-Therapie im Allgemeinen.

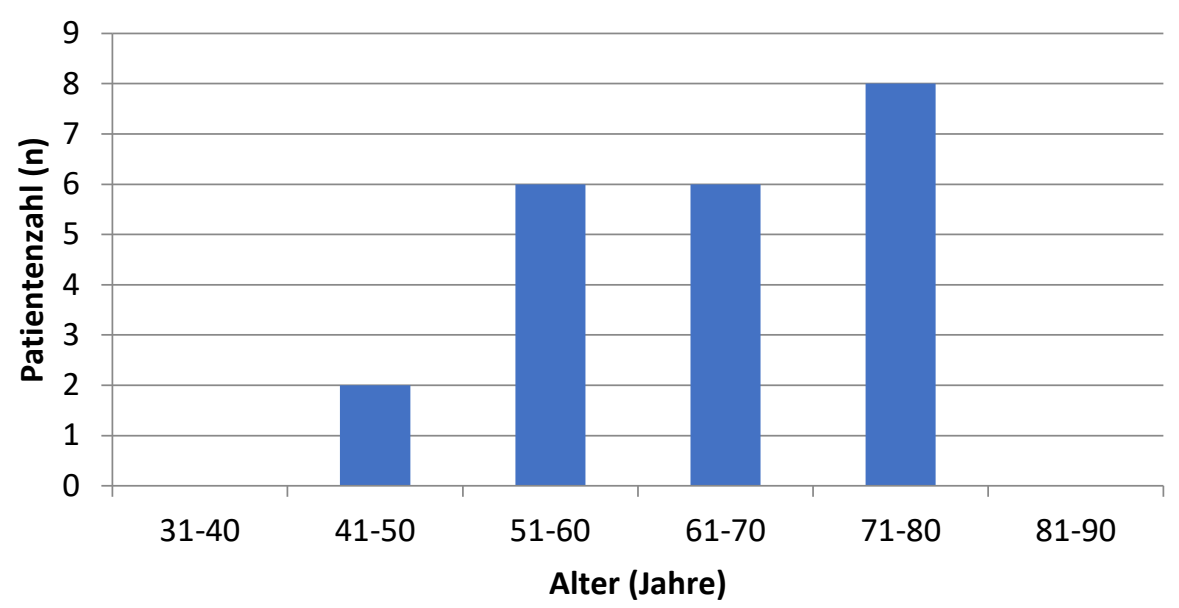

Abbildung 20: Altersverteilung der erfolgreich behandelten Patienten

Es ist anzunehmen, dass die unterschiedlichen Überlebensraten anderer Studien aus den nur eingeschränkt vergleichbaren Patientenkollektiven der einzelnen Studien resultieren. So haben zum Beispiel Park et al. Überlebensraten von $51,2 \%$ in der IABP+-Gruppe bzw. von 54,5\% in der IABP--Gruppe ermittelt. ${ }^{25}$ Ihre Studie beinhaltet jedoch weder Patienten, die älter als 80 Jahre alt sind, noch Patienten mit ernsthaften neurologischen Schädigungen oder solche mit irreversiblen Organschäden. Das mittlere Lebensalter beträgt in der IABP+Gruppe 66 Jahre und in der IABP--Gruppe 64 Jahre. Im Vergleich dazu liegt in der vorliegenden Studie das mittlere Lebensalter der IABP+-Gruppe bei 67,8 und der IABP-Gruppe bei 68,8 Jahren.

Die weitere Auswertung der Risikofaktoren hat ergeben, dass alle erfolgreich behandelten Patienten bei der Einlieferung nicht intubiert waren. Dies erlaubt den Rückschluss, dass bereits initial intubierte Patienten insgesamt ein schlechteres Outcome vorweisen werden. 76,2\% der erfolgreich behandelten Patienten haben eine relevante Herzinsuffizienz im Stadium NYHA III/IV. Dieser prozentuale Anteil an NYHA III/IV- Patienten liegt in der gleichen Größenordnung wie der aller behandelten Patienten. Ein fortgeschrittenes NYHA- Stadium lässt sich somit nicht als negativer prädiktiver Faktor identifizieren. 
Abbildung 21 vergleicht die Linksventrikuläre Ejektionsfraktion (LV-EF) und das NYHA-Stadium beider Patientenkollektive.

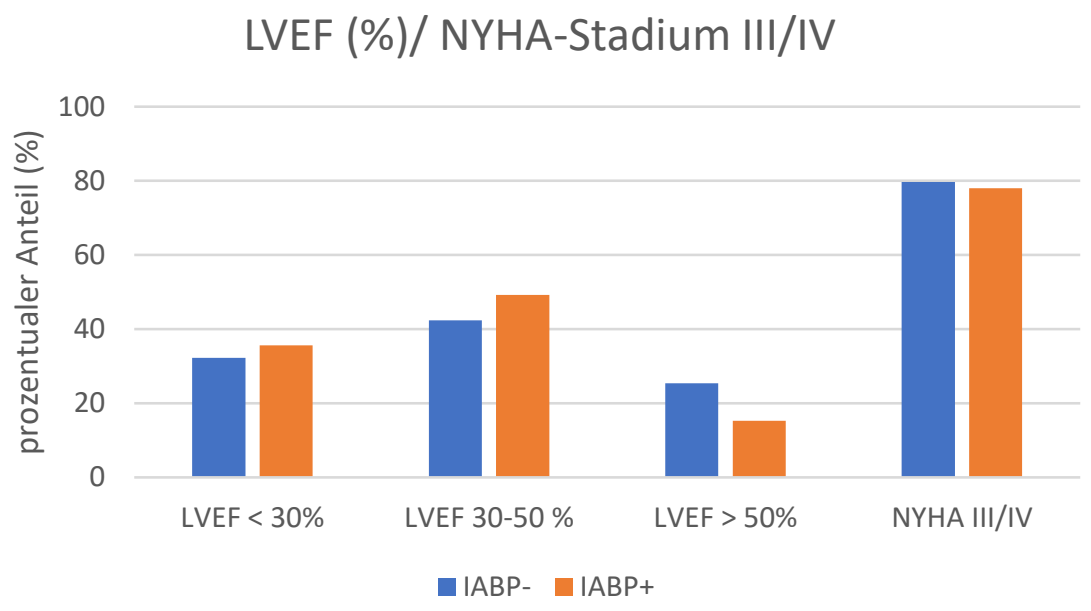

Abbildung 21: Vergleich von Linksventrikulärer-Ejektionsfraktion (LV-EF) in Prozent und NYHA-Stadium der beiden Patientenkollektive

In der Gruppe mit reiner ECLS-Therapie erlitten 40\% initial einen akuten Myokardinfarkt, 79\% verstarben im Verlauf. Im Vergleich dazu erlitten von den zusätzlich mit IABP versorgten Patienten 59\% initial einen akuten Myokardinfarkt, von innen starben $76 \%$ im Verlauf. Diese geringfügige Differenz im Hinblick auf die Mortalität nach Myokardinfarkt in beiden Patientengruppen erklärt sich möglicherweise durch die Tatsache, dass mehr Patienten bereits vor ECLSTherapie aufgrund eines akuten Myokardinfarkts eine IABP implantiert bekamen. Laut Yang et al. reduziert die zusätzliche, bereits präoperative IABP-Therapie, zumindest bei koronarchirurgischen Operationen ohne Herz-Lungen-Maschine, das Risiko für die Entwicklung eines Low-Output-Syndroms. ${ }^{16}$

Eine kardiopulmonale Reanimation vor ECLS Implantation war in der IABP-Gruppe in $25 \%$ notwendig, von denen $47 \%$ im Verlauf verstarben. In der IABP+Gruppe mussten 29\% der Patienten reanimiert werden, von innen starben $42 \%$.

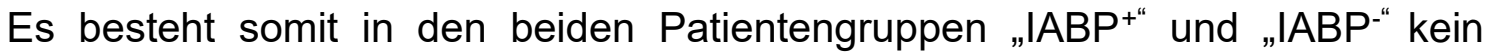
signifikanter Unterschied im Hinblick auf die Mortalität nach akutem Myokardinfarkt oder kardiopulmonaler Reanimation vor ECLS-Therapie. Abbildung 22 zeigt den prozentualen Anteil der untersuchten Faktoren in beiden Patientengruppen. 


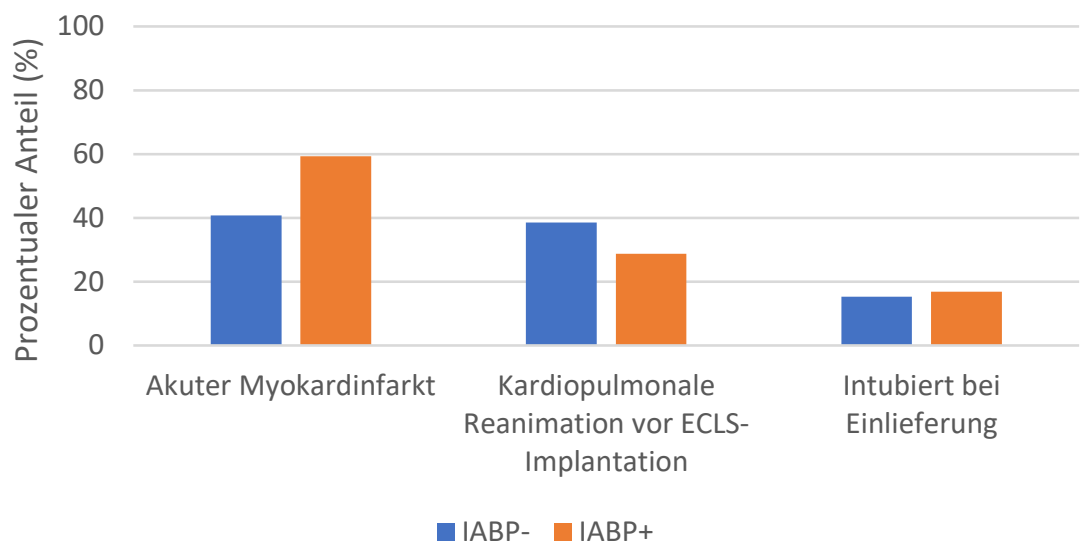

\begin{abstract}
Abbildung 22: Weitere untersuchte Faktoren in IABP+- und IABP-Gruppe im prozentualen Vergleich
\end{abstract}

\title{
4.4 Komplikationen
}

Bei der Auswertung der jeweiligen Komplikationen Rethorakotomie, Blutung an der Kanülierungsstelle, Infektion, Extremitätenischämie oder auch neurologische Komplikationen zeigt sich in allen Fällen eine Odds-Ratio von $>1$ bei nicht signifikantem P-Wert. Dennoch fällt auf, dass bei zusätzlichem Einsatz einer IABP nur ein geringerer Anteil der untersuchten Patienten eine Rethorakotomie benötigte oder bei diesen Patienten eine Blutung an der Kanülierungsstelle oder ein Nierenversagen auftrat. Es fehlen jedoch größere Studien, um möglicherweise signifikante Unterschiede festzustellen.

\subsection{Operative Eingriffe}

Bei der Auswertung der Mortalität fiel auf, dass jeder Überlebende entweder einen Koronararterienbypass mit oder ohne gleichzeitige Klappenrekonstruktionen oder eine alleinige Klappenrekonstruktionen erhalten hat. Patienten, die einen der übrigen Eingriffe erhalten haben, also eine Lungen- oder Herztransplantation, eine tracheale Rekonstruktion, die Korrektur eines Ventrikelseptumdefekts oder eine Perikardektomie, verstarben trotz ECLSTherapie. Hierbei konnte kein signifikanter Unterschied zwischen alleiniger ECLS Therapie oder zusätzlichem IABP- Einsatz nachgewiesen werden. Grund hierfür ist allem Anschein nach, die Tatsache, dass die zuletzt genannten operativen Eingriffe einen komplizierten Verlauf genommen haben und die Patienten dadurch in die Notwendigkeit einer ECLS-Therapie gekommen sind. 


\section{Zusammenfassung}

Für Patienten mit lebensbedrohlichen Herzkrankheiten ist die ExtracorporealLife-Support-Behandlung (ECLS) eine sinnvolle Therapiemöglichkeit. ${ }^{31}$ Sie bietet für Patienten im kardiogenen Schock ein Zeitfenster, um eine myokardiale Erholung zu erreichen. Hierbei kann in Abhängigkeit vom Krankheitsbild die zusätzliche Anwendung von IABP die Heilungschancen begünstigen. ${ }^{32}$ In der durchgeführten retrospektiven Studie wurden 118 Patienten betrachtet, die in der Klinik für Thorax-, Herz- und thorakale Gefäßchirurgie der Universität Frankfurt am Main im Zeitraum von Dezember 2001 bis Ende 2013 eine ECLSTherapie erhalten haben. Bei 59 Patienten wurde die ECLS-Unterstützung in Kombination mit IABP durchgeführt. Die beiden Patientenkollektive - mit und ohne IABP- sind hinsichtlich ihrer Risikofaktoren vergleichbar. Ausgehend von der Zielsetzung dieser Arbeit wurde analysiert, ob der gleichzeitige Einsatz der IABP bei ECLS - Therapie von Vor- oder sogar von Nachteil ist. Hierfür wurden für die beiden Therapiegruppen Über-lebenszeitanalysen nach Kaplan-Meier durchgeführt. Der statistische Vergleich der Überlebensraten und des Weaningerfolgs erfolgte mit Hilfe des Log-Rank-Tests.

Die Auswertung der erhobenen Daten hat ergeben, dass kein signifikanter Unterschied bei der 30-Tages-Überlebensrate und dem Weaningerfolg für die beiden Patientenkollektive mit und ohne zusätzliche IABP-Anwendung vorhanden ist. Risikofaktoren wie ein hohes Lebensalter oder eine bereits vor Einlieferung stattgefundene Intubation verringen außerdem die Überlebenschancen nach ECLS - Therapie. Ein fortgeschrittenes NYHA-Stadium konnte nicht als negativer prädiktiver Faktor identifiziert werden.

In der Literatur kommt man bezüglich der Mortalität unter alleiniger ECLSTherapie oder dem zusätzlichen Einsatz einer IABP zu unterschiedlichen Ergebnissen. Diese besagen teilweise, dass ECLS und IABP einander ergänzende Methoden sind, die sich durchaus synergistisch auf den Behandlungserfolg auswirken können und dass die Mortalität beim zusätzlichen Einsatz einer IABP signifikant niedriger ist. Die Durchführung weiterer prospektiver Studien mit vergleichbaren Patientenkollektiven zur Untersuchung des Outcomes bei den unterschiedlichen Behandlungsmethoden ist jedoch erforderlich, um ein aussagekräftiges Fazit ziehen zu können. 


\section{Executive Summary}

The Extracorporeal-Life-Support Treatment (ECLS) is a sensible therapy for patients with life-threatening heart conditions that offers patients in cardiogenic shock a time frame to achieve myocardial recovery. ${ }^{31}$ Depending on the clinical picture of the patient, the use of an Intra-aortic Balloon Pump (IABP) can improve the chances of recovery. ${ }^{32}$

This retrospective study assessed 118 patients receiving ECLS treatment at the Clinic for Thorax, Cardiac and Thoracic Vascular Surgery of the University Frankfurt am Main over a period from December 2001 until the end of 2013. Half of the patients (59) received the ECLS treatment in combination with IABP. Both patient groups - with and without IABP - were comparable with regard to their risk factors.

The objective of this work was to analyse whether the simultaneous application of IABP during the ECLS therapy would be beneficial or detrimental to the outcome of the treatment. As such, a survival analysis following the Kaplan-Meier methodology was conducted for both patient groups. Log-Rank testing allowed the statistical comparison of survival rates and the weaning success.

The analysis of the collected data showed no statistically significant difference of the 30-day survival rate and the weaning success between the two patient groups with and without the application of IABP. Furthermore, risk factors such as old age or a pre-hospital intubation reduce the chance of survival after an ECLS treatment. An advanced NYHA stage (III or IV) could not be identified as a negative predictive factor.

Literature shows variable results regarding ECLS treatment in combination with IABP. Some studies conclude that ECLS and IABP are complementary methods, which can have synergistic effects on the success of the treatment with significantly lower rates of mortality. However, further prospective studies with comparable patient groups to study the outcome with different treatment methods are necessary. 


\section{Abbildungsverzeichnis}

Abbildung 1: Schematische Darstellung des ECLS - Systems ....................... 2

Abbildung 2: Schematischer Aufbau einer Diffusionsmembran (mit freundlicher

Genehmigung der Firma Maquet (C) ........................................................ 4

Abbildung 3: Vorderseite der HU 35 (mit freundlicher Genehmigung der Firma Maquet (C) 5

Abbildung 4: Funktionsprinzip einer ROTAFLOW (C) Zentrifugalpumpe (mit freundlicher Genehmigung der Firma Maquet (C)

Abbildung 5: Kardiovaskuläre Risikofaktoren der beiden Patientenkollektive .. 16 Abbildung 6: Alters- und Geschlechtsverteilung in der IABP--Gruppe ( $n=59)$.. 21 Abbildung 7: Alters- und Geschlechtsverteilung in der IABP+-Gruppe ( $n=59) 21$

Abbildung 8: Operative Eingriffe am Gesamtkollektiv 22

Abbildung 9: Operative Eingriffe in der IABP-- und IABP+- Gruppe . 22

Abbildung 10: Prozentuale Anteile der erfolgreich entwöhnten, verstorbenen und überlebenden Patienten 23

Abbildung 11: 30-Tages-Überlebensrate mit Konfidenzbereich $\mathrm{P}=0,95$ für die IABP--Gruppe $(\mathrm{n}=59)$ 24

Abbildung 12: 30-Tages-Überlebensrate mit Konfidenzbereich $\mathrm{P}=0,95$ für die IABP+-Gruppe $(\mathrm{n}=59)$ 24

Abbildung 13: 30-Tages-Überlebensrate (1: IABP-Gruppe, 2: IABP+-Gruppe) 24 Abbildung 14: 30-Tages-Überlebensrate mit Konfidenzbereich $\mathrm{P}=0,95$ für das Gesamtkollektiv $(\mathrm{n}=118)$. 25

Abbildung 15: Todesursachen in der IABP--Gruppe ................................. 26

Abbildung 16: Todesursachen in der IABP+-Gruppe ............................... 26

Abbildung 17: Todesursachen des Gesamtkollektivs .................................. 26

Abbildung 18: Aufgetretene Komplikation ohne Einsatz einer IABP ................. 27

Abbildung 19: Aufgetretene Komplikation bei zusätzlichem Einsatz einer IABP 27

Abbildung 20: Altersverteilung der erfolgreich behandelten Patienten ............. 34 Abbildung 21: Vergleich von Linksventrikulärer-Ejektionsfraktion (LV-EF) in Prozent und NYHA-Stadium der beiden Patientenkollektive .......................... 35 Abbildung 22: Weitere untersuchte Faktoren in $\mathrm{IABP}^{+}$- und IABP--Gruppe im prozentualen Vergleich 


\section{Tabellenverzeichnis}

Tabelle 1: Stadieneinteilung der Herzinsuffizienz nach NYHA ........................ 15

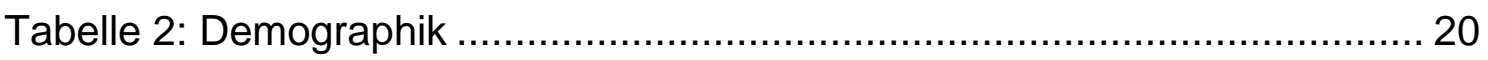

Tabelle 3: Vergleich der Überlebensraten mit anderen ECLS-Studien............. 32

Tabelle 4: Behandlungsmethode und Überlebensraten anderer Studien im

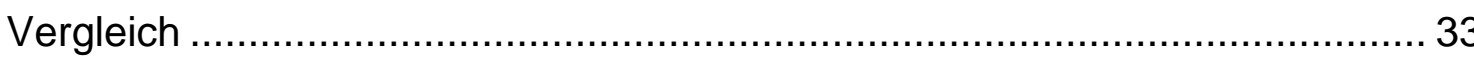




\section{Literaturverzeichnis}

References

1. Aloy A. Chirurgische Intensivmedizin: Kompendium für die Praxis. Vienna: Springer-Verlag/Wien; 2007. http://dx.doi.org/10.1007/978-3-211-29682-0.

2. Schaheen BW, Thiele RH, Isbell JM. Extracorporeal life support for adult cardiopulmonary failure. Best Pract Res Clin Anaesthesiol. 2015;29(2):229239. doi:10.1016/j.bpa.2015.04.004.

3. Palatianos GM, Foroulis CN, Vassili MI, et al. A prospective, double-blind study on the efficacy of the bioline surface-heparinized extracorporeal perfusion circuit. Ann Thorac Surg. 2003;76(1):129-135. doi:10.1016/s00034975(03)00338-2.

4. Lehle K, Philipp A, Gleich O, et al. Efficiency in extracorporeal membrane oxygenation-cellular deposits on polymethylpentene membranes increase resistance to blood flow and reduce gas exchange capacity. ASAIO J. 2008;54(6):612-617. doi:10.1097/MAT.0b013e318186a807.

5. Dornia C, Philipp A, Bauer S, et al. Visualization of thrombotic deposits in extracorporeal membrane oxygenation devices using multidetector computed tomography: a feasibility study. ASAIO J. 2013;59(4):439-441. doi:10.1097/MAT.0b013e3182976eff.

6. Ostadfar A. Biofluid Flow in Artificial, Assistive and Implantable Devices. In: Biofluid Mechanics. Elsevier; 2016:205-242.

7. Kamusella $P$, Wissgott $C$, Andresen R. Einsatz eines perkutanen Nahtverschlusssystems nach 6 - 8F transfemoralen Zugängen: Ergebnisse von 2200 Patienten. Rofo. 2012;184(4):311-315. doi:10.1055/s-00311299098.

8. Despotis G, Avidan M, Eby C. Prediction and management of bleeding in cardiac surgery. J Thromb Haemost. 2009;7 Suppl 1:111-117. doi:10.1111/j.1538-7836.2009.03412.x.

9. Natt B, Hypes C, Basken R, Malo J, Kazui T, Mosier J. Suspected HeparinInduced Thrombocytopenia in Patients Receiving Extracorporeal Membrane Oxygenation. J Extra Corpor Technol. 2017;49(1):54-58.

10.Jambor C, Bremerich D, Moritz A, Seifried E, Zwissler B. Management of hemostasis disorders after extracorporeal circulation. A clinical therapy 
algorithm. Anaesthesist. 2008;57(4):374-381. doi:10.1007/s00101-008-13368.

11. Extracorporeal Life Support Organization. General Guidelines for all ECLS Cases.

https://www.elso.org/Portals/0/ELSO\%20Guidelines\%20General\%20All\%20 ECLS\%20Version\%201_4.pdf.

12. Extracorporeal Life Support Organization. Guidelines for Adult Respiratory Failure.

https://www.elso.org/Portals/0/ELSO\%20Guidelines\%20For\%20Adult\%20Re spiratory\%20Failure\%201_4.pdf. Updated August 2017.

13.Murray JF, Matthay MA, Luce JM, Flick MR. An expanded definition of the adult respiratory distress syndrome. Am Rev Respir Dis. 1988;138(3):720723. doi:10.1164/ajrccm/138.3.720.

14.Dechert RE, Park PK, Bartlett RH. Evaluation of the oxygenation index in adult respiratory failure. J Trauma Acute Care Surg. 2014;76(2):469-473. doi:10.1097/TA.0b013e3182ab0d27.

15.Erdmann E. Klinische Kardiologie: Krankheiten des Herzens, des Kreislaufs und der herznahen Gefäße. 5., vollkommen überarbeitete und erweiterte Auflage. Berlin, Heidelberg, s.l.: Springer Berlin Heidelberg; 2000. http://dx.doi.org/10.1007/978-3-662-12156-6.

16.Yang F, Wang J, Hou D, et al. Preoperative intra-aortic balloon pump improves the clinical outcomes of off-pump coronary artery bypass grafting in left ventricular dysfunction patients. Sci Rep. 2016;6:27645. doi:10.1038/srep27645.

17.Krishna M, Zacharowski K. Principles of Intra-Aortic Balloon Pump Counterpulsation. http://www.medscape.com/viewarticle/587246_4.

18.Krishna M, Zacharowski K. Principles of Intra-Aortic Balloon Pump Counterpulsation. http://www.medscape.com/viewarticle/587246_4.

19. Ma P, Zhang Z, Song T, et al. Combining ECMO with IABP for the treatment of critically III adult heart failure patients. Heart Lung Circ. 2014;23(4):363368. doi:10.1016/j.hlc.2013.10.081.

20.Ro SK, Kim JB, Jung SH, Choo SJ, Chung CH, Lee JW. Extracorporeal life support for cardiogenic shock: influence of concomitant intra-aortic balloon 
counterpulsation. Eur J Cardiothorac Surg. 2014;46(2):186-92; discussion 192. doi:10.1093/ejcts/ezu005.

21.Unverzagt S, Machemer M-T, Solms A, et al. Intra-aortic balloon pump counterpulsation (IABP) for myocardial infarction complicated by cardiogenic shock. Cochrane Database Syst Rev. 2011;(7):CD007398. doi:10.1002/14651858.CD007398.pub2.

22.Lin L-Y, Liao C-W, Wang C-H, et al. Effects of Additional Intra-aortic Balloon Counter-Pulsation Therapy to Cardiogenic Shock Patients Supported by Extra-corporeal Membranous Oxygenation. Sci Rep. 2016;6:23838. doi:10.1038/srep23838.

23. Herold G. Innere Medizin 2013: Eine vorlesungsorientierte Darstellung ; unter Berücksichtigung des Gegenstandskataloges für die Ärztliche Prüfung ; mit ICD 10-Schlüssel im Text und Stichwortverzeichnis. Köln: Selbstverl; 2013.

24.Chang $\mathrm{C}-\mathrm{H}$, Lin $\mathrm{C}-\mathrm{Y}$, Tian $\mathrm{Y}-\mathrm{C}$, et al. Acute kidney injury classification: comparison of AKIN and RIFLE criteria. Shock. 2010;33(3):247-252. doi:10.1097/SHK.0b013e3181b2fe0c.

25.Park TK, Yang JH, Choi S-H, et al. Clinical impact of intra-aortic balloon pump during extracorporeal life support in patients with acute myocardial infarction complicated by cardiogenic shock. BMC Anesthesiol. 2014;14:27. doi:10.1186/1471-2253-14-27.

26. Aso S, Matsui H, Fushimi K, Yasunaga H. The Effect of Intraaortic Balloon Pumping Under Venoarterial Extracorporeal Membrane Oxygenation on Mortality of Cardiogenic Patients: An Analysis Using a Nationwide Inpatient Database. Crit Care Med. 2016;44(11):1974-1979. doi:10.1097/CCM.0000000000001828.

27.Li Y, Yan S, Gao S, et al. Effect of an intra-aortic balloon pump with venoarterial extracorporeal membrane oxygenation on mortality of patients with cardiogenic shock: a systematic review and meta-analysis. Eur $J$ Cardiothorac Surg. 2018. doi:10.1093/ejcts/ezy304.

28.Nuding S, Werdan K. IABP plus ECMO-Is one and one more than two? J Thorac Dis. 2017;9(4):961-964. doi:10.21037/jtd.2017.03.73.

29.Madershahian N, Liakopoulos OJ, Wippermann J, et al. The impact of intraaortic balloon counterpulsation on bypass graft flow in patients with 
peripheral ECMO. J Card Surg. 2009;24(3):265-268. doi:10.1111/j.15408191.2009.00807.x.

30.Sauren LDC, Reesink KD, Selder JL, Beghi C, van der Veen FH, Maessen JG. The acute effect of intra-aortic balloon counterpulsation during extracorporeal life support: an experimental study. Artif Organs. 2007;31(1):31-38. doi:10.1111/j.1525-1594.2007.00337.x.

31.Marinos S. Extrakorporale Membranoxygenierung und Kreislaufunterstützung als Therapie für respiratorisches und kardiales Versagen.: Risikostratifizierung und retrospektive Analyse von 200 Patienten. [Dissertation]. Frankfurt: Johann Wolfgang Goethe-Universität Frankfurt am Main; 2016.

32. Gass A, Palaniswamy C, Aronow WS, et al. Peripheral venoarterial extracorporeal membrane oxygenation in combination with intra-aortic balloon counterpulsation in patients with cardiovascular compromise. Cardiology. 2014;129(3):137-143. doi:10.1159/000365138. 


\section{Schriftliche Erklärung}

Ich erkläre ehrenwörtlich, dass ich die dem Fachbereich Medizin der Johann Wolfgang Goethe - Universität Frankfurt am Main zur Promotionsprüfung eingereichte Dissertation mit dem Titel

Extracorporeal Life Support in Kombination mit IABP. Behandlungsmöglichkeiten und Grenzen einer supportiven Therapie bei kardialem Versagen. Retrospektive Analyse von 118 Patienten.

in der Klinik für Thorax-, Herz- und Thorakale Gefäßchirurgie unter Betreuung und Anleitung von Prof. Dr. Nestoras Papadopoulos mit Unterstützung durch Dr. Spiros Marinos ohne sonstige Hilfe selbst durchgeführt und bei der Abfassung der Arbeit keine anderen als die in der Dissertation angeführten Hilfsmittel benutzt habe. Darüber hinaus versichere ich, nicht die Hilfe einer kommerziellen Promotionsvermittlung in Anspruch genommen zu haben.

Ich habe bisher an keiner in- oder ausländischen Universität ein Gesuch um Zulassung zur Promotion eingereicht. Die vorliegende Arbeit wurde bisher nicht als Dissertation eingereicht. 